\title{
SMITH FORMS OF PALINDROMIC MATRIX POLYNOMIALS*
}

\author{
D. STEVEN MACKEY ${ }^{\dagger}$, NILOUFER MACKEY ${ }^{\dagger}$, CHRISTIAN MEHL ${ }^{\ddagger}$, AND VOLKER \\ MEHRMANN ${ }^{\ddagger}$
}

\begin{abstract}
Many applications give rise to matrix polynomials whose coefficients have a kind of reversal symmetry, a structure we call palindromic. Several properties of scalar palindromic polynomials are derived, and together with properties of compound matrices, used to establish the Smith form of regular and singular $T$-palindromic matrix polynomials over arbitrary fields. The invariant polynomials are shown to inherit palindromicity, and their structure is described in detail. Jordan structures of palindromic matrix polynomials are characterized, and necessary conditions for the existence of structured linearizations established. In the odd degree case, a constructive procedure for building palindromic linearizations shows that the necessary conditions are sufficient as well. The Smith form for *-palindromic polynomials is also analyzed. Finally, results for palindromic matrix polynomials over fields of characteristic two are presented.
\end{abstract}

Key words. Compound matrix, Elementary divisors, Invariant polynomials, Jordan structure, Matrix pencil, Matrix polynomial, Palindromic matrix polynomial, Smith form, Structured linearization.

AMS subject classifications. 65F15, 15A18, 15A21, 15A54, 15A57.

1. Introduction. Polynomial eigenvalue problems arise in many applications, and often the underlying matrix polynomial is structured in some way. Of particular interest are matrix polynomials $P(\lambda)=\sum_{i=0}^{k} \lambda^{i} A_{i}$ that have palindromic structure. Coefficients of such polynomials have symmetry under certain involutions: $A_{i} \mapsto \varepsilon A_{k-i}^{T}$ in the $T$-palindromic case, or $A_{i} \mapsto \varepsilon A_{k-i}^{*}$ in the $*$-palindromic case, where $\varepsilon= \pm 1$. $T$-palindromic polynomials arise in the vibrational analysis of railroad tracks excited by high speed trains [11,20], and in the modelling and numerical simulation of periodic surface acoustic wave (SAW) filters [12, 28]. Complex *-palindromic polynomials occur when the Crawford number of two Hermitian matrices is computed [10], as well as in the solution of discrete-time linear-quadratic optimal control problems via structured eigenvalue problems [2].

*Received by the editors on May 15, 2010. Accepted for publication on November 23, 2010. Handling Editor: Bit-Shun Tam.

${ }^{\dagger}$ Department of Mathematics, Western Michigan University, Kalamazoo, MI 49008, USA (steve.mackey@wmich.edu, nil.mackey@wmich.edu). Supported by National Science Foundation grants DMS-0713799 and DMS-1016224. Support from Deutsche Forschungsgemeinschaft through DFG Research Center MATHEON during research visits to TU Berlin is gratefully acknowledged.

${ }^{\ddagger}$ Institut für Mathematik, MA 4-5, Technische Universität Berlin, 10623 Berlin, Germany (mehl@math.tu-berlin.de, mehrmann@math.tu-berlin.de). Supported by Deutsche Forschungsgemeinschaft through DFG Research Center Matheon, 'Mathematics for key technologies' in Berlin. 
The standard computational approach to solving polynomial eigenproblems is by linearization. However, when the original polynomial is structured, it can be both theoretically and numerically advantageous to use a linearization with the same structure, thereby preserving any spectral symmetries that may be present.

Linearizations that reflect the structure of regular palindromic polynomials were introduced in [21], where sufficient criteria for the existence of strong palindromic linearizations were presented, along with a systematic method for constructing them. Recall that a matrix polynomial $P(\lambda)$ is regular if $\operatorname{det} P(\lambda) \not \equiv 0$, whereas $P(\lambda)$ is said to be singular if either $\operatorname{det} P(\lambda) \equiv 0$, or $P(\lambda)$ is not square. The analysis in [21] revealed a disparity between $T$-palindromic and $*$-palindromic structure which arises even in the $1 \times 1$ case, as the following example illustrates.

Example 1.1. ([21]). Consider the palindromic $1 \times 1$ matrix polynomial $P(\lambda)=$ $\lambda^{2}-1$. A $T$-palindromic linearization for $P(\lambda)$ would be a $2 \times 2$ matrix polynomial of the form $L_{\varepsilon}(\lambda)=\lambda Z+\varepsilon Z^{T}$ where $\varepsilon= \pm 1$, and with +1 and -1 as its only eigenvalues, each with multiplicity one. This means $Z$ would have to be invertible, and thus $\operatorname{det}\left(\varepsilon Z^{-1} Z^{T}\right)=\varepsilon^{2} \operatorname{det}\left(Z^{-1} Z^{T}\right)=(\operatorname{det} Z)^{-1} \operatorname{det} Z=1$. But $\varepsilon Z^{-1} Z^{T}$ has only the simple eigenvalues +1 and -1 , so $\operatorname{det}\left(\varepsilon Z^{-1} Z^{T}\right)=-1$, a contradiction. On the other hand, a $*$-palindromic linearization would have the form $L_{\varepsilon}(\lambda)=\lambda Z+\varepsilon Z^{*}$, and the same argument, mutatis mutandis, yields no contradiction, since now $\operatorname{det}\left(\varepsilon Z^{-1} Z^{*}\right)$ may lie anywhere on the unit circle in the complex plane and so can be equal to -1 .

In fact, it was proved in [21] that all regular *-palindromic polynomials have palindromic linearizations. A complete study of the Jordan structures of $*$-palindromic polynomials can thus be carried out in the regular case by exploiting their structured linearizations, as was done by Lancaster, Prells and Rodman in [17] for *palindromic polynomials with nonsingular leading coefficient. Investigating the Jordan structures of $T$-palindromic polynomials and characterizing which polynomials admit palindromic linearizations remained an open problem even for the regular case, while the singular case was not considered at all.

It is these issues that we turn to in this paper, using Smith forms and compound matrices as the key tools. The structured linearization question is completely settled for all odd degree $T$-palindromic polynomials, both regular and singular, while the even degree case is settled only for regular $T$-palindromics over the real and complex fields. We do this by following the path in [22], where the analogous questions were investigated for $T$-alternating matrix polynomials, i.e., matrix polynomials $P$ satisfying $P(-\lambda)= \pm P^{T}(\lambda)$. Although the overall strategy in this paper is quite similar to the one in [22], the palindromic case shows some fundamental differences which require a separate treatment of the subject. In particular, a key fact in [22] was the observation that the Euclidean algorithm for computing the greatest common divisor of scalar polynomials preserves $T$-alternating structure, since whenever 
two $T$-alternating scalar polynomials are divided, then the remainder and quotient are also $T$-alternating. Unfortunately, the corresponding statements are not true in the case of $T$-palindromic polynomials, leading to subtle differences in the theories of alternating and palindromic scalar polynomials. Also, in [22] necessary and sufficient conditions for a Smith form to be that of a $T$-alternating matrix polynomial could be given. The $T$-palindromic case turns out to be more complicated, as shown in Section 7.

The outline of the remainder of the paper is as follows. In Section 2, we review some facts from matrix theory used in the proofs of the main results. In Section 4, the earlier definition of palindromic matrix polynomials from [21] is extended to allow zero leading coefficients. This requires a more careful consideration of the degree and the reversal of matrix polynomials, a task that is performed in Section 3. Key ingredients needed for the proofs of the main theorems are results on scalar palindromic polynomials and the structure of determinants and minors of palindromic matrix polynomials, developed in Sections 5 and 6, respectively. Section 7 then presents one of the main results of this paper, laying out necessary conditions for a Smith form to be that of a $T$-palindromic matrix polynomial over an arbitrary field of characteristic different from two. For completeness, the simpler case of the Smith form of $*$-palindromic polynomials over the complex field is also analyzed. Section 8 characterizes the possible Jordan structures of $T$-palindromic matrix polynomials, which lead in turn to necessary conditions for a $T$-palindromic matrix polynomial to have a $T$-palindromic linearization in Section 9. In the odd-degree case, these conditions turn out to be sufficient as well. It is worth pointing out that most results apply to singular as well as regular T-palindromic matrix polynomials. Finally, the Smith form for palindromic polynomials over fields of characteristic two is discussed in Appendix A.

2. Notation and matrix theory tools. Throughout the paper we use $\mathbb{N}$ to denote the set of non-negative integers, $\mathbb{F}$ to denote an arbitrary field, and $\mathbb{F}[x]$ to denote the ring of polynomials in one variable with coefficients from the field $\mathbb{F}$. Matrix polynomials are of the form $P(\lambda)=\sum_{i=0}^{k} \lambda^{i} A_{i}$, where $A_{0}, \ldots, A_{k} \in \mathbb{F}^{m \times n}$. Equivalently, $P(\lambda)$ can also be viewed as a matrix with polynomial entries. Some wellknown tools and results from matrix theory that we need are now briefly reviewed. Detailed proofs can be found in standard monographs like [7, Ch.VI], [9, Part IV], [19].

2.1. Smith form and partial multiplicity sequences. Recall that two $m \times n$ matrix polynomials $P(\lambda), Q(\lambda)$ are called equivalent if there exists unimodular matrix polynomials $E(\lambda)$ and $F(\lambda)$ of size $m \times m$ and $n \times n$, respectively, such that

$$
Q(\lambda)=E(\lambda) P(\lambda) F(\lambda)
$$


Here, an $n \times n$ matrix polynomial $E(\lambda)$ is called unimodular if $\operatorname{det} E(\lambda)$ is a nonzero constant, independent of $\lambda$.

ThEOREM 2.1 (Smith form $[6]$ ). Let $P(\lambda)$ be an $m \times n$ matrix polynomial over an arbitrary field $\mathbb{F}$. Then there exists $r \in \mathbb{N}$, and unimodular matrix polynomials $E(\lambda)$ and $F(\lambda)$ of size $m \times m$ and $n \times n$, respectively, such that

$$
E(\lambda) P(\lambda) F(\lambda)=\operatorname{diag}\left(d_{1}(\lambda), \ldots, d_{\min \{m, n\}}(\lambda)\right)=: D(\lambda)
$$

where $d_{1}(\lambda), \ldots, d_{r}(\lambda)$ are monic (i.e., the highest degree terms all have coefficient 1$)$, $d_{r+1}(\lambda), \ldots, d_{\min \{m, n\}}(\lambda)$ are identically zero, and $d_{1}(\lambda), \ldots, d_{r}(\lambda)$ form a divisibility chain, that is, $d_{j}(\lambda)$ is a divisor of $d_{j+1}(\lambda)$ for $j=1, \ldots, r-1$. Moreover, $D(\lambda)$ is unique.

The nonzero diagonal elements $d_{j}(\lambda)$ for $j=1, \ldots, r$ in the Smith form of $P(\lambda)$ are called the invariant factors or invariant polynomials of $P(\lambda)$ and have an important interpretation in terms of greatest common divisors of minors of $P(\lambda)[7,9,19]$. Recall that a minor of order $k$ of an $m \times n$ matrix $A$ is the determinant of a $k \times k$ submatrix of $A$, i.e., of a matrix obtained from $A$ by deleting $m-k$ rows and $n-k$ columns.

For $d(x) \neq 0$, we write $d(x) \mid p(x)$ to mean that $d(x)$ is a divisor of $p(x)$. When $S$ is a set of scalar polynomials, we write $d(x) \mid S$ to mean that $d(x)$ divides each element of $S$, i.e., $d(x)$ is a common divisor of the elements of $S$. The greatest common divisor (or GCD) of a set $S$ containing at least one nonzero polynomial is the unique monic polynomial $g(x)$ such that $g(x) \mid S$, and $d(x) \mid S$ implies $d(x) \mid g(x)$.

TheOREM 2.2 (Characterization of invariant polynomials). Let $P(\lambda)$ be an $m \times n$ matrix polynomial over an arbitrary field $\mathbb{F}$ with Smith form as in $(2.2)$. Set $p_{0}(\lambda) \equiv 1$. For $1 \leq j \leq \min \{m, n\}$, let $p_{j}(\lambda) \equiv 0$ if all minors of $P(\lambda)$ of order $j$ are zero; otherwise, let $p_{j}(\lambda)$ be the greatest common divisor $(G C D)$ of all minors of $P(\lambda)$ of order $j$. Then the number $r$ in Theorem 2.1 is the largest integer such that $p_{r}(\lambda) \not \equiv 0$, i.e., $r=\operatorname{rank} P(\lambda)$. Furthermore, the invariant polynomials $d_{1}(\lambda), \ldots, d_{r}(\lambda)$ of $P(\lambda)$ are ratios of $G C D$ s given by

$$
d_{j}(\lambda)=\frac{p_{j}(\lambda)}{p_{j-1}(\lambda)}, \quad j=1, \ldots, r
$$

while the remaining diagonal entries of the Smith form of $P(\lambda)$ are given by

$$
d_{j}(\lambda)=p_{j}(\lambda) \equiv 0, \quad j=r+1, \ldots, \min \{m, n\} .
$$

Definition 2.3 (Partial multiplicity sequences). Let $P(\lambda)$ be an $m \times n$ matrix polynomial of rank $r$ over a field $\mathbb{F}$. For any $\lambda_{0} \in \mathbb{F}$, the invariant polynomials $d_{i}(\lambda)$ 
of $P$ for $1 \leq i \leq r$ can each be uniquely factored as

$$
d_{i}(\lambda)=\left(\lambda-\lambda_{0}\right)^{\alpha_{i}} q_{i}(\lambda) \text { with } \quad \alpha_{i} \geq 0, q_{i}\left(\lambda_{0}\right) \neq 0 .
$$

The sequence of exponents $\left(\alpha_{1}, \alpha_{2}, \ldots, \alpha_{r}\right)$ satisfies the condition $0 \leq \alpha_{1} \leq \alpha_{2} \leq$ $\cdots \leq \alpha_{r}$ by the divisibility chain property of the Smith form, and is called the partial multiplicity sequence of $P$ at $\lambda_{0}$.

Note that this sequence is allowed to consist of $r$ zeroes; in fact this happens for all but a finite number of elements of $\mathbb{F}$. We say $\lambda_{0} \in \mathbb{F}$ is a (finite) eigenvalue of $P$ whenever its partial multiplicity sequence $\left(\alpha_{1}, \alpha_{2}, \ldots, \alpha_{r}\right)$ is not the zero sequence. The elementary divisors for an eigenvalue $\lambda_{0}$ of $P$ are the collection of factors $(\lambda-$ $\left.\lambda_{0}\right)^{\alpha_{i}}$ with $\alpha_{i}>0$, including repetitions. Observe that the algebraic multiplicity of an eigenvalue $\lambda_{0}$ is just the sum of the terms in its partial multiplicity sequence, $\alpha_{1}+\alpha_{2}+\cdots+\alpha_{r}$, while the geometric multiplicity is simply the number of nonzero terms in this sequence. Matrix polynomials may also have infinite eigenvalues; these will be defined and discussed in Section 3, along with the notion of the elementary divisors at $\infty$. The spectrum of $P$ is the collection of all (finite and infinite) eigenvalues of $P$. It is worth noting that the definition of the spectrum of a matrix polynomial via the Smith form subsumes the more restrictive notion of the spectrum as the roots of $\operatorname{det} P(\lambda)$; using det $P(\lambda)$ is completely inadequate for singular matrix polynomials, and also misses the possibility of $\infty$ as an eigenvalue altogether.

2.2. Compound matrices and their properties. Compound matrices were used in [22] to obtain Smith forms of $T$-even and $T$-odd matrix polynomials. They will also play an important role in establishing the main results in this paper. For references on compound matrices, see [13, Section 0.8], [23, Chapter I.2.7], [24, Sections 2 and 28]. We use a variation of the notation in [13] for submatrices of an $m \times n$ matrix $A$. Let $\eta \subseteq\{1, \ldots, m\}$ and $\kappa \subseteq\{1, \ldots, n\}$ be arbitrary index sets of cardinality $j \leq \min \{m, n\}$. Then $A_{\eta \kappa}$ denotes the $j \times j$ submatrix of $A$ in rows $\eta$ and columns $\kappa$, and the $\eta \kappa$-minor of order $j$ of $A$ is $\operatorname{det} A_{\eta \kappa}$. Note that $A$ has $\left(\begin{array}{c}m \\ j\end{array}\right) \cdot\left(\begin{array}{c}n \\ j\end{array}\right)$ minors of order $j$.

Definition 2.4 (Compound matrices). Let $A$ be an $m \times n$ matrix with entries in an arbitrary commutative ring, and let $\ell \leq \min \{m, n\}$ be a positive integer. Then the $\ell$ th compound matrix (or the $\ell$ th adjugate) of $A$, denoted $\mathcal{C}_{\ell}(A)$, is the $\left(\begin{array}{c}m \\ \ell\end{array}\right) \times\left(\begin{array}{l}n \\ \ell\end{array}\right)$ matrix whose $(\eta, \kappa)$-entry is the $\ell \times \ell$ minor $\operatorname{det} A_{\eta \kappa}$ of $A$. Here, the index sets $\eta \subseteq\{1, \ldots, m\}$ and $\kappa \subseteq\{1, \ldots, n\}$ of cardinality $\ell$ are ordered lexicographically.

Observe that we always have $\mathcal{C}_{1}(A)=A$, and, if $A$ is square, $\mathcal{C}_{n}(A)=\operatorname{det} A$. Basic properties of $\mathcal{C}_{\ell}(A)$ are listed in the next theorem.

TheOREM 2.5 (Properties of compound matrices). Let $A$ be an $m \times n$ matrix with entries in a commutative ring, and let $\ell \leq \min \{m, n\}$ be a positive integer. Then 

(a) $\mathcal{C}_{\ell}\left(A^{T}\right)=\left(\mathcal{C}_{\ell}(A)\right)^{T}$;
(b) $\mathcal{C}_{\ell}(\mu A)=\mu^{\ell} \mathcal{C}_{\ell}(A)$, where $\mu \in \mathbb{F}$;
(c) $\operatorname{det} \mathcal{C}_{\ell}(A)=(\operatorname{det} A)^{\beta}$, where $\beta=\left(\begin{array}{c}n-1 \\ \ell-1\end{array}\right)$, provided that $m=n$;
(d) $\mathcal{C}_{\ell}(A B)=\mathcal{C}_{\ell}(A) \mathcal{C}_{\ell}(B)$, provided that $B \in \mathbb{F}^{n \times p}$ and $\ell \leq \min (m, n, p)$.

We are especially interested in compounds of matrices with polynomial entries. Note that such a compound can be thought of either as a polynomial with matrix coefficients, or as a matrix with polynomial entries, leading to the natural identification $\mathcal{C}_{\ell}(P)(\lambda):=\mathcal{C}_{\ell}(P(\lambda))$. The next theorem, established in [22], shows how the first $\ell+1$ invariant polynomials of $P(\lambda)$ determine the first two invariant polynomials of $C_{\ell}(P)(\lambda)$.

Theorem 2.6 (First two invariant polynomials of the $\ell$ th compound [22]). Suppose the Smith form of an $n \times n$ matrix polynomial $P(\lambda)$ is

$$
D(\lambda)=\operatorname{diag}\left(d_{1}(\lambda), \ldots, d_{\ell-1}(\lambda), d_{\ell}(\lambda), d_{\ell+1}(\lambda), \ldots, d_{n}(\lambda)\right)
$$

where $2 \leq \ell<n$. If $C(\lambda)=\left(c_{1}(\lambda), c_{2}(\lambda), \ldots, c_{\left(\begin{array}{c}n \\ \ell\end{array}\right)}(\lambda)\right)$ is the Smith form of $\mathcal{C}_{\ell}(P)(\lambda)$, then the first two invariant polynomials of $\mathcal{C}_{\ell}(P)(\lambda)$ are given by

$$
c_{1}(\lambda)=d_{1}(\lambda) \cdots d_{\ell-1}(\lambda) d_{\ell}(\lambda) \quad \text { and } \quad c_{2}(\lambda)=d_{1}(\lambda) \cdots d_{\ell-1}(\lambda) d_{\ell+1}(\lambda) .
$$

3. Reversal operator revisited. From now on we consider only square matrix polynomials $P(\lambda)=\sum_{i=0}^{k} \lambda^{i} A_{i}$, where $A_{k}, \ldots, A_{0} \in \mathbb{F}^{n \times n}$ with $\mathbb{F}$ an arbitrary field, but we allow leading coefficient matrices to be zero as well as $P$ to be singular. Consequently, we have to ensure that eigenvalues and their multiplicities are welldefined. Consider, for example, the polynomial

$$
P(\lambda)=A=\lambda 0_{n}+A,
$$

with $A \in \mathbb{F}^{n \times n}$ nonsingular. As we shall see, the spectrum of $P(\lambda)$ depends on whether $P(\lambda)$ is being viewed as $A$ or as $\lambda 0_{n}+A$. It must therefore be clarified whether $P(\lambda)$ is to be viewed as a constant matrix or a matrix pencil, or even as a matrix polynomial of higher "degree". We do this by specifying the grade of a matrix polynomial, which is an integer at least as large as its degree. (This notion was considered by Gohberg, Kaashoek and Lancaster in [8], and called "extended degree" in [16].) Clearly, a polynomial of grade $k$ can also be viewed as a polynomial of any grade higher than $k$, so the grade under consideration must be made explicit. When the grade is not specified, we assume that it is the same as the degree. Note that the grade of a matrix polynomial $P(\lambda)$ constitutes an aspect of $P(\lambda)$ in addition to its degree. It does not replace the notion of degree, which retains its usual meaning as the largest $m$ such that the coefficient of $\lambda^{m}$ in $P(\lambda)$ is nonzero. 
EXAMPLE 3.1. The ordered list of coefficients of an $n \times n$ matrix polynomial of degree 1 and grade 3 has the form $\left(A_{3}, A_{2}, A_{1}, A_{0}\right)=\left(0_{n}, 0_{n}, A_{1}, A_{0}\right)$, with $A_{1} \neq 0_{n}$.

The term pencil will be reserved for matrix polynomials of grade one, and they will usually be denoted by $L(\lambda)$. Most numerical methods for solving polynomial eigenproblems first turn the matrix polynomial into a pencil via linearization, which we now define. While the classical definition in [9] involves the degree of the matrix polynomial, allowing leading coefficient matrices to be zero means that linearizations must now be defined with respect to the grade of the polynomial.

DEFinition 3.2 (Linearization). Let $P(\lambda)$ be a nonzero $n \times n$ matrix polynomial of grade $k \geq 1$. Then a matrix pencil $L(\lambda)=\lambda X+Y$ with $X, Y \in \mathbb{F}^{k n \times k n}$ is a linearization of $P(\lambda)$ with respect to grade $k$ if there exist unimodular matrix polynomials $E(\lambda), F(\lambda)$ such that

$$
E(\lambda) L(\lambda) F(\lambda)=\left[\begin{array}{c|c}
P(\lambda) & 0 \\
\hline 0 & I_{(k-1) n}
\end{array}\right] .
$$

A linearization $L$ of $P$ has the same eigenvalues as $P$, but the partial multiplicity sequences of $L$ and $P$ at the eigenvalue infinity (see Definition 3.4 below) may differ. Hence a more restrictive notion of linearization was introduced in [8] and called strong linearization in [18], where it was shown that ordinary linearizations in the sense of Definition 3.2 can have Jordan structure at $\infty$ that is arbitrarily different (except for equal algebraic multiplicity) from that of $P$. Though considered only for regular matrix polynomials in $[8,18]$, the notion of strong linearization is applicable to singular polynomials as well. To define these concepts, we begin with the notion of reversal.

Definition 3.3 ( $j$-reversal). Let $P$ be a nonzero matrix polynomial of degree $d \geq 0$. For $j \geq d$, the $j$-reversal of $P$ is the matrix polynomial $\operatorname{rev}_{j} P$ given by

$$
\left(\operatorname{rev}_{j} P\right)(\lambda):=\lambda^{j} P(1 / \lambda) \text {. }
$$

In the special case when $j=d$, the $j$-reversal of $P$ is called the reversal of $P$ and denoted by rev $P$.

Definition 3.4 (Infinite eigenvalues). Let $P$ be a nonzero matrix polynomial of grade $k$. We say $\lambda_{0}=\infty$ is an eigenvalue of $P$ whenever 0 is an eigenvalue of $\operatorname{rev}_{k} P$, and the partial multiplicity sequence of $P$ at $\lambda_{0}=\infty$ is defined to be the same as that of the eigenvalue 0 for $\operatorname{rev}_{k} P$. The elementary divisors of $\lambda_{0}=\infty$ are the same as those of the eigenvalue 0 of $\operatorname{rev}_{k} P$.

ExAmple 3.5. Let $A \in \mathbb{F}^{n \times n}$ be invertible. Then the constant polynomial $P(\lambda)=A$ of grade 0 has no eigenvalues - there are no finite eigenvalues because $P(\lambda)$ has a trivial Smith form (all its invariant polynomials are 1), and since $\left(\operatorname{rev}_{0} P\right)(\lambda)=$ 
$A$, the invertibility of $A$ means that $P$ does not have $\infty$ as an eigenvalue either. On the other hand when viewed as the matrix pencil $P(\lambda)=\lambda 0_{n}+A$, we have $\left(\operatorname{rev}_{1} P\right)(\lambda)=\lambda A+0_{n}$. Since $A \in \mathbb{F}^{n \times n}$ is invertible, $\operatorname{rev}_{1} P$ has the eigenvalue 0 with algebraic multiplicity $n$. Thus when viewed as a pencil, $P$ has no finite eigenvalues, but it does have the eigenvalue $\infty$ with algebraic multiplicity $n$.

Definition 3.6 (Strong linearization [8]). Let $P(\lambda)$ be a nonzero matrix polynomial of grade $k \geq 1$ and degree $d \geq 0$. A matrix pencil $L(\lambda)$ is called a strong linearization for $P(\lambda)$ if $L(\lambda)$ is a linearization for $P(\lambda)$, and $\left(\operatorname{rev}_{1} L\right)(\lambda)$ is a linearization for $\left(\operatorname{rev}_{k} P\right)(\lambda)$.

Any strong linearization $L(\lambda)$ of a regular polynomial $P(\lambda)$ preserves the partial multiplicity sequences of all eigenvalues of $P(\lambda)$, both finite and infinite [8].

It is well known that elementary divisors are closely related to the Jordan block structure of matrices, and to the Jordan chain structure of regular matrix polynomials. For example, there is a one-to-one correspondence between the elementary divisors of $\lambda I_{n}-A$ and the Jordan blocks of the matrix $A \in \mathbb{C}^{n \times n}$. More generally, the degrees of the elementary divisors of a matrix polynomial $P(\lambda)$ over the field $\mathbb{C}$ correspond to the lengths of the Jordan chains of $P(\lambda)$; see [9] or [22] for further details. Therefore for a matrix polynomial $P$ over a field $\mathbb{F}$, we will refer to the collection of all the finite and infinite elementary divisors of $P$, including repetitions, when $P$ is viewed as a polynomial over the algebraic closure $\overline{\mathbb{F}}$, as the Jordan structure of $P$. Clearly, the Jordan structure can be read from the partial multiplicity sequences of the finite and infinite eigenvalues of $P$.

We end this section with some observations on $j$-reversals, and two lemmas that list some useful properties. Taking the reversal puts the coefficients of $P$ in reverse order without increasing the degree. Taking the $j$-reversal can be viewed as a kind of "shifted reversal", and the degree may increase, decrease, or stay the same. For example, the 2-reversal of the matrix polynomial of degree one $P(\lambda)=\lambda A_{1}+A_{0}$, where $A_{0} \neq 0$, is $\operatorname{rev}_{2} P(\lambda)=\lambda^{2} A_{0}+\lambda A_{1}$, a polynomial of higher degree. Taking the 2-reversal again now lowers the degree to one, and brings us back to $P$, illustrating an involutory property that holds in general.

Lemma 3.7 (Properties of reversals). Let $P$ be a nonzero matrix polynomial, and $j \geq \operatorname{deg} P$.

(a) $\operatorname{deg}(\operatorname{rev} P) \leq \operatorname{deg} P$ and $\operatorname{deg}\left(\operatorname{rev}_{j} P\right) \leq j$, with equality in either case if and only if $P(0) \neq 0$.

(b) $\operatorname{rev}(\operatorname{rev} P)=P \Leftrightarrow P(0) \neq 0$.

(c) $\operatorname{rev}_{j}\left(\operatorname{rev}_{j} P\right)=P$.

(d) $\left(\operatorname{rev}_{j} P\right)(\lambda)=\lambda^{j-k}(\operatorname{rev} P)(\lambda)$, where $k=\operatorname{deg} P$. 
Proof. The proof follows directly from the defining equation (3.3) of $j$-reversals. $\square$

Note that in Lemma 3.7 there is no contradiction between parts (b) and (c), even though (b) has the condition $P(0) \neq 0$ and (c) does not (taking $j=\operatorname{deg} P$ in (c)). The key difference between (b) and (c) is that $\operatorname{deg}(\operatorname{rev} P)$ might not be the same as $\operatorname{deg} P$, and so the second rev operation in (b) may not be the same as the second $\mathrm{rev}_{j}$ operation in (c), as illustrated in the following example.

ExAmple 3.8. Let $P(\lambda)=\lambda^{2} A_{2}+\lambda A_{1}$, where $A_{2} \neq 0_{n}, A_{1} \neq 0_{n}$. Since $\operatorname{deg} P=2$, we have $(\operatorname{rev} P)(\lambda)=\left(\operatorname{rev}_{2} P\right)(\lambda)=\lambda A_{1}+A_{2}$, which is a polynomial of degree 1. This means $(\operatorname{rev}(\operatorname{rev} P))(\lambda)=\operatorname{rev}_{1}\left(\lambda A_{1}+A_{2}\right)=\lambda A_{2}+A_{1} \neq P(\lambda)$. On the other hand, $\left(\operatorname{rev}_{2}\left(\operatorname{rev}_{2} P\right)\right)(\lambda)=\operatorname{rev}_{2}\left(\lambda A_{1}+A_{2}\right)=\lambda^{2} A_{2}+\lambda A_{1}=P(\lambda)$.

We remark that Lemma 3.7 will be particularly useful in Section 5 when applied to ordinary scalar polynomials. Another result that will be needed is the multiplicative property of reversals of scalar polynomials. The proof follows directly from the definition of reversal and so is omitted.

LEMMA 3.9 (Reversals of products). Let $p(x)$ and $q(x)$ be scalar polynomials, and let $j \geq \operatorname{deg} p, \ell \geq \operatorname{deg} q$. Then $\operatorname{rev}_{(j+\ell)}(p q)=\operatorname{rev}_{j} p \cdot \operatorname{rev}_{\ell} q$. In particular, with $j=\operatorname{deg} p$ and $\ell=\operatorname{deg} q$, we have $\operatorname{rev}(p q)=\operatorname{rev} p \cdot \operatorname{rev} q$.

Extending Lemma 3.9 to matrix polynomials requires additional hypotheses, as the product of nonzero matrices may be zero. We will not need such results in this paper.

4. Palindromic matrix polynomials. Many applications, several of which are summarized in $[2,20,21]$, give rise to matrix polynomials with $T$-palindromic structure. We now generalize the definition introduced in [20].

Definition 4.1 (Palindromic). A nonzero $n \times n$ matrix polynomial $P$ of degree $d \geq 0$ is said to be $T$-palindromic if $\left(\operatorname{rev}_{j} P\right)(\lambda)= \pm P^{T}(\lambda)$ for some integer $j$, with $j \geq d$.

By allowing $j$ to be larger than the degree of the polynomial, and permitting a sign change, this definition extends the notion of palindromicity from the usage established in $[20,21]$, where $T$-palindromic polynomials were only those satisfying $(\operatorname{rev} P)(\lambda)=P^{T}(\lambda)$. Extending the definition as we have done here helps to formulate the results of this paper with greater clarity and conciseness.

Now if $P$ is $T$-palindromic, what can be said about the admissible values of $j$ in Definition 4.1? Consider the following example.

ExAmple 4.2. $P(\lambda)=\lambda^{2} A-\lambda A^{T}$ is $T$-palindromic, since $\left(\operatorname{rev}_{3} P\right)(\lambda)=-P^{T}(\lambda)$. 
Observe that $j=3$ is the only grade that will work for $P(\lambda)$ in Example 4.2. The next lemma establishes the uniqueness of $j$ in general; this unique $j$ will be referred to as the grade of palindromicity of $P(\lambda)$.

Proposition 4.3 (Uniqueness of the grade of palindromicity). Let $P(\lambda)$ be a nonzero T-palindromic matrix polynomial. Then there exists a unique nonnegative integer $j$ such that $\left(\operatorname{rev}_{j} P\right)(\lambda)= \pm P^{T}(\lambda)$. Furthermore, if $P(\lambda)=\lambda^{\ell} Q(\lambda)$ where $\ell \in \mathbb{N}$, and $Q(0) \neq 0$, then $j=\ell+\operatorname{deg} P=2 \ell+\operatorname{deg} Q$.

Proof. By hypothesis, there exists $j \geq m=\operatorname{deg} P$ such that

$$
\left(\operatorname{rev}_{j} P\right)(\lambda)= \pm P^{T}(\lambda)
$$

Since $P(\lambda)=\lambda^{\ell} Q(\lambda)$ with $Q(0) \neq 0$, we conclude $\operatorname{deg} P=\ell+\operatorname{deg} Q$, with the lowest nonzero coefficient of $P$ being $A_{\ell}$. Forming the $j$-tuple of coefficients of $P$ we get

$$
\left(A_{j}, \ldots, A_{m}, \ldots, A_{\ell}, \ldots, A_{0}\right)=\left(0_{n \times n}, \ldots, 0_{n \times n}, A_{m}, \ldots, A_{\ell}, 0_{n \times n}, \ldots, 0_{n \times n}\right) .
$$

By (4.1), the number $\ell$ of trailing zero matrices must be the same as the number of leading zero matrices, which is $j-m$. Thus $\ell=j-\operatorname{deg} P$, and substituting $\operatorname{deg} P=\ell+\operatorname{deg} Q$, we get $j=\ell+\operatorname{deg} P=2 \ell+\operatorname{deg} Q$ is uniquely determined.

Clearly, a nonzero $T$-palindromic polynomial over a field $\mathbb{F}$ with char $\mathbb{F} \neq 2$ cannot simultaneously satisfy $\operatorname{rev}_{j} P=P^{T}$ and $\operatorname{rev}_{j} P=-P^{T}$, and thus the following notion is well-defined.

Definition 4.4 (Palindromic type). The palindromic type $\varepsilon(P)$ of a nonzero $T$-palindromic matrix polynomial $P$ over a field $\mathbb{F}$ with char $\mathbb{F} \neq 2$ is defined by

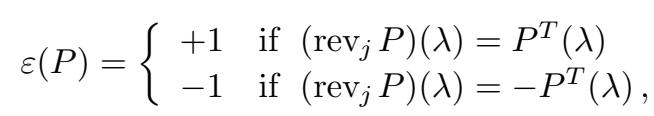

where $j$ is the grade of palindromicity.

We will abbreviate palindromic type to type for expediency. The expression ' $P$ is $T$-palindromic with grade of palindromicity $j$ ' will usually be abbreviated to ' $P$ is $T$-palindromic of grade $j$ '. This signifies that the grade of $P$ as well as the grade of palindromicity of $P$ is $j$. Thus, for example, the polynomial $\lambda^{2} A-\lambda A^{T}$ is $T$ palindromic of grade 3 . When $j=\operatorname{deg} P$, we sometimes say ' $P$ is $T$-palindromic with respect to degree'. If $\varepsilon(P)=-1$, it may sometimes be convenient to say ' $P$ is $T$-anti-palindromic', following the usage in [21]. By convention, every zero matrix polynomial is $T$-palindromic with respect to all grades $j \in \mathbb{N}$.

The next corollary addresses the effect of removing all $\lambda$-factors from a palindromic polynomial. 
Corollary 4.5. Let $P(\lambda)=\lambda^{\ell} Q(\lambda)$ be a nonzero T-palindromic polynomial, where $\ell \in \mathbb{N}$, and $Q(0) \neq 0$. Then $Q$ is T-palindromic with respect to degree, of the same type as $P$.

Proof. By (4.2), we see that the tuple of coefficients of $Q$ is just $\left(A_{m}, \ldots, A_{\ell}\right)$. The result now follows directly from the $T$-palindromicity of $P$.

Theorem 4.6 (Compounds of palindromic matrix polynomials). If $P$ is an $n \times n$ $T$-palindromic matrix polynomial of grade $k$ and $1 \leq r \leq n$, then the rth compound of $P$ is also T-palindromic, of grade $k r$ and type $(\varepsilon(P))^{r}$.

Proof. Using the properties of compound matrices given in Theorem 2.5(a)(b), we have

$$
\begin{aligned}
P^{T}(\lambda)=\varepsilon(P) \lambda^{k} P(1 / \lambda) & \Longrightarrow \mathcal{C}_{r}\left(P^{T}(\lambda)\right)=\mathcal{C}_{r}\left(\varepsilon(P) \lambda^{k} P(1 / \lambda)\right) \\
& \Longrightarrow\left(\mathcal{C}_{r}(P(\lambda))\right)^{T}=(\varepsilon(P))^{r} \lambda^{k r} \mathcal{C}_{r}(P(1 / \lambda)) \\
& \Longrightarrow\left(\mathcal{C}_{r}(P)(\lambda)\right)^{T}=(\varepsilon(P))^{r}\left(\operatorname{rev}_{k r}\left(\mathcal{C}_{r}(P)\right)\right)(\lambda),
\end{aligned}
$$

from which the desired result is immediate.

5. Palindromic scalar polynomials. Palindromic scalar polynomials play an important role in the investigation of possible Smith forms for $T$-palindromic matrix polynomials. For a nonzero polynomial $p(x)$ in $\mathbb{F}[x]$, the reversal operations $\left(\operatorname{rev}_{j} p\right)(x):=x^{j} p(1 / x)$, and $(\operatorname{rev} p)(x)$ are just a special case of Definition 3.3 , and so palindromic scalar polynomials can be defined, mutatis mutandis, via Definition 4.1 for matrix polynomials. Since the transpose operation is now superfluous, the terminology can be simplified.

Definition 5.1 (Palindromic scalar polynomials). A nonzero polynomial $p(x)$ in $\mathbb{F}[x]$ is palindromic if $\operatorname{rev}_{j} p= \pm p$ for some integer $j$, with $j \geq \operatorname{deg} p$.

As before, we can say ' $p$ is palindromic of grade $j$ ' to specify that $j$ is the unique grade of palindromicity, as well as the grade of $p$. Because this arises frequently in the context of scalar polynomials, we will instead use the simpler abbreviation ' $p$ is $j$-palindromic'. Every nonzero constant polynomial is 0-palindromic, and, by convention, the zero polynomial is palindromic with respect to all grades $j \in \mathbb{N}$. The type of palindromicity, $\varepsilon(p)$, is defined in accordance with Definition 4.4.

EXAMPLE 5.2. Let $P(\lambda)$ be a nonzero $T$-palindromic matrix polynomial of grade $k$. Then the diagonal entries of $P(\lambda)$, which in general may be of varying individual degrees, are all $k$-palindromic scalar polynomials of the same type as $P(\lambda)$.

Section 5.1 establishes some of the basic algebraic properties of palindromic scalar polynomials. A number of these properties are quite elementary, and have surely 
appeared repeatedly in the literature. One recent example is the popular expository book [15]; see also the references therein.

5.1. Products, quotients, factorizations. It will be useful to know when palindromicity is preserved under multiplication and division, and to describe a factorization of scalar palindromic polynomials into irreducibles. For alternating (i.e., even or odd) matrix polynomials, the strategy in [22] hinged upon a key fact: if $p(x)$ and $d(x)$ are alternating scalar polynomials with $\operatorname{deg} d \leq \operatorname{deg} p$, then the division $p(x)=q(x) d(x)+r(x)$ always yields an alternating quotient $q(x)$ and an alternating remainder $r(x)$.

Unfortunately, palindromicity is not preserved under division: when the palindromic $p(x)=x^{3}+x^{2}+x+1$ is divided by the palindromic $d(x)=x^{2}+3 x+1$, neither the quotient $q(x)=x-2$ nor the remainder $r(x)=6 x+3$ is palindromic. Here is what we can say.

Lemma 5.3 (Products and quotients). Let $p, q \in \mathbb{F}[x]$ be nonzero polynomials such that $p$ is $j$-palindromic and $q$ is $k$-palindromic.

(a) If $j<k$, then $p$ is not divisible by $q$.

(b) The product $p q$ is $(j+k)$-palindromic of type $\varepsilon(p) \varepsilon(q)$.

(c) If $q$ divides $p$, then $j \geq k$ and the quotient $\frac{p(x)}{q(x)}$ is $(j-k)$-palindromic of type $\varepsilon(p) \varepsilon(q)$.

Proof. (a): Write $p(x)=x^{\ell} p_{1}(x), q(x)=x^{m} q_{1}(x)$, with $p_{1}(0) \neq 0, q_{1}(0) \neq 0$, and $\ell, m \in \mathbb{N}$. If $\ell<m$, the result follows immediately. When $\ell \geq m$, by Proposition 4.3 we have $j=2 \ell+\operatorname{deg} p_{1}$, and $k=2 m+\operatorname{deg} q_{1}$. Now $j<k$ and $\ell \geq m$ implies

$$
\operatorname{deg} p_{1}<\operatorname{deg} q_{1} .
$$

Thus $p_{1}$ cannot be divisible by $q_{1}$, and hence $p$ is not divisible by $q$ either.

(b), (c): The proofs are immediate from the definitions of palindromicity and of reversals, and from the additive property of the degrees of scalar polynomials under multiplication and division.

Lemma 5.4 (Reversals of irreducibles). Suppose $d(x) \in \mathbb{F}[x]$ is nonconstant and irreducible over $\mathbb{F}$, and $d(x) \neq x$. Then $\operatorname{rev} d(x)$ is also nonconstant and irreducible over $\mathbb{F}$. Moreover, the product $p(x)=d(x) \cdot \operatorname{rev} d(x)$ is $j$-palindromic of type +1 , with $j=\operatorname{deg} p=2 \operatorname{deg} d$.

Proof. Since $d(x)$ is irreducible and $d(x) \neq x$, we have $d(0) \neq 0$, so $\operatorname{deg}(\operatorname{rev} d)=$ $\operatorname{deg} d$ and $\operatorname{rev}(\operatorname{rev} d)=d$ by Lemma 3.7. Now suppose that rev $d$ was not irreducible, and had a nontrivial factorization $\operatorname{rev} d(x)=r(x) s(x)$. Then $r$ and $s$ are both polynomials of degree at least 1 with $r(0) \neq 0$ and $s(0) \neq 0$, since $\operatorname{rev} d(0) \neq 0$. Taking 
rev of both sides of the equation $\operatorname{rev} d(x)=r(x) s(x)$, we see that

$$
d(x)=\operatorname{rev}(r(x) s(x))=\operatorname{rev} r(x) \operatorname{rev} s(x)
$$

displays a nontrivial factorization of $d(x)$, contradicting its irreducibility. The calculation

$$
\operatorname{rev} p(x)=\operatorname{rev}(d(x) \cdot \operatorname{rev} d(x))=\operatorname{rev} d(x) \cdot \operatorname{rev}(\operatorname{rev} d(x))=\operatorname{rev} d(x) \cdot d(x)=p(x)
$$

shows that $p(x)$ is palindromic with respect to degree, of type +1 . Since $\operatorname{deg}(\operatorname{rev} d)=$ $\operatorname{deg} d$, we have $j=\operatorname{deg} p=2 \operatorname{deg} d$.

LemMa 5.5 (Irreducible factors of palindromic polynomials). Suppose $p(x) \in \mathbb{F}[x]$ is palindromic with respect to degree and $d(x)$ is a nonconstant irreducible factor of $p(x)$. Then $\operatorname{revd}(x)$ is also a nonconstant irreducible factor of $p(x)$. If $d(x)$ is not palindromic, then $d(x)$ and revd $(x)$ are distinct irreducibles (i.e., relatively prime irreducibles), and the product $f(x)=d(x) \cdot \operatorname{rev} d(x)$ is a $j$-palindromic factor of $p(x)$ of type +1 , where $j=\operatorname{deg} f=2 \operatorname{deg} d$.

Proof. If $p(x)=d(x) q(x)$, then

$$
p(x)= \pm \operatorname{rev} p(x)= \pm \operatorname{rev}(d(x) q(x))= \pm \operatorname{rev} d(x) \operatorname{rev} q(x)
$$

shows that $\operatorname{rev} d(x)$ is also a factor of $p(x)$. Since $p(x)$ is palindromic with respect to degree, we have $p(0) \neq 0$ by Proposition 4.3 , and hence $d(x) \neq x$. Thus rev $d(x)$ is a nonconstant irreducible factor of $p(x)$ by Lemma 5.4. The irreducible factors $d(x)$ and $\operatorname{rev} d(x)$ will be distinct as long as $d(x) \neq \alpha \operatorname{rev} d(x)$ for any constant $\alpha$. But $d(x)=\alpha \operatorname{rev} d(x)$ occurs if and only if $\operatorname{rev} d(x)=\operatorname{rev}(\alpha \operatorname{rev} d(x))=\alpha d(x)=$ $\alpha^{2} \operatorname{rev} d(x)$ if and only if $\alpha^{2}=1$, or in other words if and only if $d(x)$ is palindromic. Thus when $d(x)$ and $\operatorname{rev} d(x)$ are distinct irreducibles, then both are factors of $p(x)$, so that $f(x)=d(x) \cdot \operatorname{rev} d(x)$ is a palindromic factor of $p(x)$. That $f(x)$ is palindromic with respect to degree and of type +1 , with $\operatorname{deg} f=2 \operatorname{deg} d$, follows from Lemma 5.4 .

Observe that there are only two monic irreducible palindromic polynomials of degree 1 , viz., $(x+1)$ and $(x-1)$. Unless char $\mathbb{F}=2$, these are distinct polynomials, and they play a special role in the analysis of Smith forms of $T$-palindromic matrix polynomials. For the rest of this section, we assume char $\mathbb{F} \neq 2$, and establish factorizations for palindromic scalar polynomials over such fields. We will use the convention that the parity of an integer $j$ is $(-1)^{j}$.

Lemma 5.6 (Presence of $(x+1),(x-1)$ factors). Let $q \in \mathbb{F}[x]$ be a nonzero $j$-palindromic polynomial, where char $\mathbb{F} \neq 2$.

(a) If $\varepsilon(q)$ and the parity of $j$ have opposite sign, i.e., if $(-1)^{j} \varepsilon(q)=-1$, then $(x+1)$ is a factor of $q$. 
(b) If $\varepsilon(q)=-1$, then $(x-1)$ is a factor of $q$.

Proof. (a): Evaluating $x^{j} q(1 / x)=\varepsilon(q) q(x)$ at $x=-1$ gives us $(-1)^{j} q(-1)=$ $\varepsilon(q) q(-1)$. Since $(-1)^{j} \varepsilon(q)=-1$, we have $q(-1)=0$, i.e., $(x+1)$ is a factor of $q$. (b): Evaluating $x^{j} q(1 / x)=-q(x)$ at $x=1$ immediately yields the desired result.

Lemma 5.7 (Multiplicity of $(x+1)$ and $(x-1)$ ). Let $p(x) \in \mathbb{F}[x]$ be a nonzero $j$-palindromic polynomial, where char $\mathbb{F} \neq 2$.

(a) The multiplicity of $(x+1)$ as a factor of $p$ has

(i) the same parity as $j$, if $\varepsilon(p)=1$.

(ii) the opposite parity as $j$, if $\varepsilon(p)=-1$.

(b) The multiplicity of $(x-1)$ as a factor of $p$ is independent of the palindromic grade $j$, and its parity has the same value as $\varepsilon(p)$.

Proof. (a): Write $p(x)=(x+1)^{\ell} q(x)$, where $q(-1) \neq 0$, so $(x+1)$ is not a factor of $q$. Now by Lemma $5.3(\mathrm{~b}),(x+1)^{\ell}$ is $\ell$-palindromic of type +1 for any $\ell \in \mathbb{N}$. Hence by Lemma $5.3(\mathrm{c}), q(x)$ is $(j-\ell)$-palindromic of the same type as $p(x)$.

Now consider the case when $\varepsilon(p)=1$. Then $\varepsilon(q)=1$. If $j$ and $\ell$ have opposite parity, then $k:=j-\ell$ is odd, so $(-1)^{k} \varepsilon(q)=-1$. By Lemma 5.6(a), $q$ must have $(x+1)$ as a factor, a contradiction. A similar argument establishes (ii).

(b): Write $p(x)=(x-1)^{k} q(x)$, where $q(1) \neq 0$, so $(x-1)$ is not a factor of $q$. Now $(x-1)^{k}$ is palindromic by Lemma $5.3(\mathrm{~b})$, and hence so is $q$ by Lemma $5.3(\mathrm{c})$. As $(x-1)$ is not a factor of $q$, we conclude by Lemma $5.6(\mathrm{~b})$ that $\varepsilon(q)=1$. This means that the palindromic types of $p$ and $(x-1)^{k}$ must agree, by Lemma $5.3(\mathrm{c})$. Thus $\varepsilon(p)=\varepsilon\left((x-1)^{k}\right)=(\varepsilon(x-1))^{k}=(-1)^{k}$, as desired.

Theorem 5.8 (Irreducible palindromic factorization). A polynomial $p(x) \in \mathbb{F}[x]$ with char $\mathbb{F} \neq 2$ is palindromic if and only if it admits an irreducible factorization in $\mathbb{F}[x]$ of the form

$$
p(x)=c_{0} \cdot x^{k_{1}}(x-1)^{k_{2}}(x+1)^{k_{3}} \cdot \prod_{i=1}^{\mu} b_{i}(x)^{m_{i}} \cdot \prod_{j=1}^{\kappa}\left(c_{j} d_{j}(x) \operatorname{rev} d_{j}(x)\right)^{n_{j}}
$$

where $c_{0}, c_{1}, \ldots, c_{\kappa} \in \mathbb{F}$ are nonzero, $k_{1}, k_{2}, k_{3} \in \mathbb{N}, m_{1}, \ldots, m_{\mu}, n_{1}, \ldots, n_{\kappa} \in \mathbb{N}$ are nonzero, and the irreducible factors $b_{i}(x), d_{j}(x)$ and $\operatorname{rev} d_{j}(x)$ are distinct with the following properties:

(a) Each $b_{i}(x)$ and each $d_{j}(x)$ is monic with $b_{i}(0) \neq 0, b_{i}( \pm 1) \neq 0$, and $d_{j}(0) \neq 0$, $d_{j}( \pm 1) \neq 0$. The nonzero constants $c_{j} \in \mathbb{F}$ are chosen so that $c_{j} \operatorname{rev} d_{j}(x)$ is also monic, for $j=1, \ldots, \kappa$.

(b) Each $b_{i}(x)$ has even degree, with $\operatorname{deg} b_{i} \geq 2$, and each $b_{i}(x)$ is palindromic with respect to degree, of type $\varepsilon\left(b_{i}\right)=1$. 
(c) The factors $d_{j}(x)$ and $\operatorname{rev} d_{j}(x)$ are not palindromic, and may have any degree, with $\operatorname{deg} d_{j}=\operatorname{deg} \operatorname{rev} d_{j} \geq 1$.

The parities of $k_{2}$ and $k_{3}$ are as stated in Lemma 5.7. The factorization (5.1) is unique up to reordering of the $b_{i}(x)$ 's amongst themselves, the $d_{j}(x)$ 's amongst themselves, and exchanging the roles of $d_{j}(x)$ and $c_{j} \operatorname{rev} d_{j}(x)$ within each $d_{j}(x)$, rev $d_{j}(x)$ pair.

Proof. $\Leftarrow$ : If $p$ has a factorization of the form (5.1), then $p$ is palindromic by Lemma 5.3(b).

$\Rightarrow$ : Write $p(x)=c_{0} x^{k_{1}}(x-1)^{k_{2}}(x+1)^{k_{3}} q(x)$ where $c_{0} \in \mathbb{F}$ is nonzero, $k_{1}, k_{2}, k_{3} \in$ $\mathbb{N}, q(x)$ is monic, and $q(0) \neq 0, q( \pm 1) \neq 0$. Then $q$ is palindromic by Lemma $5.3(\mathrm{c})$, and $q(0) \neq 0$ implies that $q$ must be palindromic with respect to its degree by Corollary 4.5 .

Since $(x-1)$ is not a factor of $q$, we must have $\varepsilon(q)=1$ by Lemma $5.6(\mathrm{~b})$. Likewise, every palindromic factor of $q$ must also be of type +1 . In addition, no factor of $q$ can vanish at $x=0, \pm 1$, since $q$ is not zero at these values.

The proof proceeds by induction on the degree of the monic, palindromic polynomial $q$ of type +1 . If $\operatorname{deg} q=0$, then we are done. Otherwise, $\operatorname{deg} q \geq 2$, since $(x+1)$ is the only monic palindromic of degree 1 and type +1 . Let $d(x)$ be a monic irreducible factor of $q(x)$.

If $d(x)$ is palindromic, then $q(x)=d(x) \widetilde{q}(x)$, where $\widetilde{q}$ is palindromic by Lemma $5.3(\mathrm{c})$. Since $(x-1)$ is not a factor of $\widetilde{q}$, we must have $\varepsilon(\widetilde{q})=1$ by Lemma $5.6(\mathrm{~b})$, and therefore, $\varepsilon(d)=1$. Now $d$ is palindromic with respect to its degree, since $d(0) \neq 0$. If $\operatorname{deg} d$ were odd, then by Lemma 5.6(a), $(x+1)$ would be a factor of $d$, since $\varepsilon(d)=1$. Hence $\operatorname{deg} d$ is even. Note also that because $q$ and $d$ are monic, so is $\widetilde{q}$.

On the other hand, if $d(x)$ is not palindromic, then $q(x)=d(x) \operatorname{rev} d(x) \widehat{q}(x)$, by Lemma 5.5. Here $\widehat{q}$ is palindromic and of type $\varepsilon(\widehat{q})=1$ by Lemma 5.3(c). Now rev $d$ may not be monic. Choose $c \in \mathbb{F}$ so that $c$ is nonzero and $c(\operatorname{rev} d)$ is monic. Then we have $q(x)=d(x) \cdot c \operatorname{rev} d(x) \cdot \frac{1}{c} \widehat{q}(x)$. Since $q, d$ and $c(\operatorname{rev} d)$ are all monic, $\widetilde{q}:=\frac{1}{c} \widehat{q}$ is also monic. Since $\widehat{q}$ is of type +1 , so is $\widetilde{q}$.

Thus in both cases (whether $d$ is palindromic or not), we can apply the inductive hypothesis to the monic palindromic polynomial $\widetilde{q}(x)$ of type +1 , yielding the desired factorization.

Finally we note that the factorization (5.1) implies that the polynomial $p$ is $\left(k_{1}+\right.$ $\operatorname{deg} p$ )-palindromic. The parities of $k_{2}$ and $k_{3}$ now follow from Lemma 5.7. Since the factorization of an arbitrary polynomial in $\mathbb{F}[x]$ into monic irreducibles is unique up to reordering of the factors, the uniqueness of the factorization (5.1) up to the described reordering is immediate. 
If the underlying field is algebraically closed, then combining the previous results yields the following factorization of palindromic polynomials.

COROLlary 5.9 (Factorization of palindromic polynomials). Let $\mathbb{F}$ be an algebraically closed field with char $\mathbb{F} \neq 2$. A polynomial $p(x) \in \mathbb{F}[x]$ is palindromic if and only if $p$ can be expressed as a product of linear factors of the form

$$
p(x)=c x^{k_{1}}(x+1)^{k_{2}}(x-1)^{k_{3}} \prod_{i=1}^{m}\left(\left(x-\lambda_{i}\right)\left(x-\lambda_{i}^{-1}\right)\right)^{\ell_{i}}
$$

where $c \in \mathbb{F} \backslash\{0\}, k_{1}, k_{2}, k_{3}, \ell_{1}, \ldots, \ell_{m} \in \mathbb{N}$, and where $\lambda_{1}, \ldots, \lambda_{m}, \lambda_{1}^{-1}, \ldots, \lambda_{m}^{-1} \in$ $\mathbb{F} \backslash\{0\}$ are distinct. Moreover, the parities of $k_{2}$ and $k_{3}$ are as stated in Lemma 5.7.

5.2. GCDs. It is standard notation to write $d \mid p$ to mean that the polynomial $d$ divides the polynomial $p$. Here we extend that notation to a set $S$ of polynomials, and write $d \mid S$ to mean that $d$ divides each element of $S$, or in other words that $d$ is a common divisor of the elements of $S$. The greatest common divisor (GCD) of a set $S$ containing at least one nonzero polynomial is then the unique monic polynomial $\operatorname{gcd}(S)$ satisfying $\operatorname{gcd}(S) \mid S$ and $q|S \Rightarrow q| \operatorname{gcd}(S)$. We follow the convention that the GCD of the set consisting of only the zero polynomial is the zero polynomial.

Proposition 5.10 (GCD of palindromic polynomials). Let $S$ be a nonempty, finite set of palindromic polynomials over an arbitrary field $\mathbb{F}$. Then $\operatorname{gcd}(S)$ is palindromic. (The polynomials in $S$ may be of varying degrees, and they may be palindromic with respect to different grades and have different types.)

Proof. If $S$ contains only one polynomial, then clearly we are done. Otherwise, let $S=\left\{p_{1}(x), p_{2}(x), \ldots, p_{r}(x)\right\}$ where each $p_{i}$ is palindromic. We prove the result by induction on $\max \operatorname{deg} p_{i}$.

If $\max \operatorname{deg} p_{i}=0$, then clearly $\operatorname{gcd}(S)=1$, which is palindromic. Next, make the inductive hypothesis that $\operatorname{gcd}(S)$ is palindromic for every finite set $S$ of palindromic polynomials $p_{i}$ with $\max \operatorname{deg} p_{i} \leq n$.

Now consider a finite set $S$ of palindromic polynomials $p_{i}$ such that $\max \operatorname{deg} p_{i}=$ $n+1$. If $\operatorname{gcd}(S)=1$ then we are done. Otherwise, let $d(x)$ be an irreducible common factor of $S$, of degree at least one. By Lemma 5.5 we know that either $d(x)$ itself is palindromic, or $d(x) \cdot \operatorname{rev} d(x)$ is a palindromic common factor of $S$. Letting $a(x)$ denote $d(x)$ in the first case, or $d(x) \cdot \operatorname{rev} d(x)$ in the second case, we have $S=\left\{a(x) q_{1}(x), a(x) q_{2}(x), \ldots, a(x) q_{r}(x)\right\}$ where each $q_{i}(x)$ is palindromic by Lemma 5.3(c), and $\operatorname{deg} q_{i}(x) \leq n$. Thus

$$
\operatorname{gcd}(S)=a(x) \operatorname{gcd}\left\{q_{1}(x), q_{2}(x), \ldots, q_{r}(x)\right\}=a(x) b(x)
$$


where $b(x)$ is palindromic by the induction hypothesis. Hence $\operatorname{gcd}(S)$ is palindromic by Lemma $5.3(\mathrm{~b})$, and the induction is complete.

Another notion that will be useful is that of reversal pairs.

Definition 5.11 (Reversal pairs). Nonzero polynomials $p, q \in \mathbb{F}[x]$ form a $j$-reversal pair if $\operatorname{rev}_{j} p= \pm q$ for some integer $j$ with $j \geq \max \{\operatorname{deg} p, \operatorname{deg} q\}$.

Observe that the $j$-reversal pair relation is symmetric in $p$ and $q$, since $\operatorname{rev}_{j} p= \pm q$ if and only if $\operatorname{rev}_{j} q= \pm p$, by Lemma $3.7(\mathrm{c})$.

EXAMPLE 5.12. In a $T$-palindromic matrix polynomial $P(\lambda)$ of grade $j$, the off-diagonal entries $P(\lambda)_{i k}$ and $P(\lambda)_{k i}$ always form a $j$-reversal pair of scalar polynomials.

LEMmA 5.13 (Characterization of reversal pairs). Let $p, q, s \in \mathbb{F}[x]$ be nonzero polynomials such that $p(x)=x^{\ell} q(x)$ with $q(0) \neq 0$. Then the following are equivalent:

(a) $p$ and $s$ form a $j$-reversal pair.

(b) $s(x)= \pm x^{k} \operatorname{rev} q(x)$ with $k=j-\ell-\operatorname{deg} q \geq 0$.

Proof. Note that $p(x)$ and $s(x)$ form a $j$-reversal pair if and only if

$$
s(x)= \pm \operatorname{rev}_{j} p(x)= \pm x^{j} p(1 / x)= \pm x^{j} x^{-\ell} q(1 / x)= \pm x^{j-\ell-\operatorname{deg} q} \operatorname{rev} q(x) .
$$

Hence, the result follows.

The GCD of a $j$-reversal pair of scalar polynomials turns out to be palindromic, a fact which will have a key influence on the GCD of minors of a palindromic matrix polynomial. We first establish a special case.

Lemma 5.14. Let $q \in \mathbb{F}[x]$, with $q(0) \neq 0$. Then $\operatorname{gcd}\{q(x), \operatorname{rev} q(x)\}$ is palindromic.

Proof. If $q$ and $\operatorname{rev} q$ are relatively prime, then we are done, since any constant polynomial is clearly palindromic. Otherwise, $q$ and $\operatorname{rev} q$ have at least one factor in common. Choose $d(x)$, an irreducible common factor of degree at least 1 . We can therefore write

$$
\begin{aligned}
q(x) & =d(x) w(x), \\
\operatorname{rev} q(x) & =d(x) v(x) .
\end{aligned}
$$

Note that every factor of $q$ has a nonzero constant term since $q(0) \neq 0$. Taking reversals of both equations (5.3) and using Lemmas 3.9 and 3.7(b) we obtain

$$
\begin{aligned}
\operatorname{rev} q(x) & =\operatorname{rev} d(x) \operatorname{rev} w(x), \\
q(x) & =\operatorname{rev} d(x) \operatorname{rev} v(x),
\end{aligned}
$$


revealing that $\operatorname{rev} d(x)$ is also a common factor of $q$ and $\operatorname{rev} q$. If $d(x)$ is palindromic, then $\operatorname{rev} d(x)= \pm d(x)$ and so from (5.3a) and (5.4a) we conclude

$$
\operatorname{gcd}\{q(x), \operatorname{rev} q(x)\}=d(x) \operatorname{gcd}\{w(x), \operatorname{rev} w(x)\} .
$$

If $d(x)$ is not palindromic, we need to find an analog to (5.5). As $d(x)$ is irreducible with $d(0) \neq 0$, by Lemma 5.4, $\operatorname{rev} d(x)$ is also irreducible. Since $d(x)$ and $\operatorname{rev} d(x)$ are distinct irreducible factors of $q$, we can factor the term $w(x)$ in (5.3a), and then use the reversal to get

$$
\begin{aligned}
q(x) & =d(x) \operatorname{rev} d(x) \widetilde{w}(x), \\
\operatorname{rev} q(x) & =\operatorname{rev} d(x) d(x) \operatorname{rev} \widetilde{w}(x),
\end{aligned}
$$

thus obtaining the desired analog of (5.5)

$$
\operatorname{gcd}\{q(x), \operatorname{rev} q(x)\}=d(x) \operatorname{rev} d(x) \operatorname{gcd}\{\widetilde{w}(x), \operatorname{rev} \widetilde{w}(x)\} .
$$

Now repeat this argument. With the role of $q(x)$ being played by $w(x)$ or $\widetilde{w}(x)$, as appropriate, each iteration produces a new palindromic factor of $p:=\operatorname{gcd}\{q, \operatorname{rev} q\}$ until the GCD on the right-hand-side of (5.5) or (5.7) is 1. At this point, $p$ has been expressed as a product of palindromic factors (in at $\operatorname{most} \operatorname{deg}(p)$ iterations). Since the product of palindromic polynomials is again palindromic, the proof is complete. $\square$

Proposition 5.15 (GCD of reversal pairs). The GCD of a j-reversal pair of nonzero polynomials is palindromic.

Proof. Let $p(x)$ and $s(x)$ form a $j$-reversal pair. Then, by Lemma 5.13 , there exists $q(x)$ with $q(0) \neq 0$ such that $p(x)=x^{m} q(x)$ and $s(x)= \pm x^{k} \operatorname{rev} q(x)$ for some $m, k \geq 0$. Thus we have $\operatorname{gcd}\{p(x), s(x)\}=x^{\mu} \operatorname{gcd}\{q(x), \operatorname{rev} q(x)\}$, where $\mu=\min \{k, m\}$. Now $x^{\mu}$ is $2 \mu$-palindromic, and so the result follows by Lemma 5.14 and Lemma 5.3(b).

6. Determinants and minors. As Theorem 2.2 shows, the minors of a matrix polynomial are intimately connected with its Smith form. We begin with the $n \times n$ minor, i.e., the determinant of $P(\lambda)$, which can be directly shown to inherit palindromicity.

Proposition 6.1 (Determinant of $T$-palindromic polynomials). If $P(\lambda)$ is an $n \times n$ T-palindromic polynomial of grade $k$, then $\operatorname{det} P(\lambda)$ is a kn-palindromic polynomial. If $P(\lambda)$ is regular, then $\varepsilon(\operatorname{det} P)=(\varepsilon(P))^{n}$.

Proof. For any $n \times n$ polynomial $P(\lambda)$ of grade $k$ we have $k n \geq \operatorname{deg}(\operatorname{det} P)$. Since $P$ is $T$-palindromic,

$$
\begin{aligned}
\operatorname{det} P(\lambda) & =\operatorname{det}\left[\varepsilon(P) \lambda^{k} P^{T}(1 / \lambda)\right] \\
& =(\varepsilon(P))^{n} \lambda^{k n} \operatorname{det} P(1 / \lambda) \\
& =(\varepsilon(P))^{n}\left(\operatorname{rev}_{k n}(\operatorname{det} P)\right)(\lambda)
\end{aligned}
$$


from which the desired result immediately follows.

Recall that $A_{\eta \kappa}$ denotes the $\ell \times \ell$ submatrix of an $m \times n$ matrix $A$ in rows and columns $\eta$ and $\kappa$ where $\eta \subseteq\{1, \ldots, m\}$, and $\kappa \subseteq\{1, \ldots, n\}$ are subsets of cardinality $\ell \leq \min \{m, n\}$. Submatrices of $A$ and $A^{T}$ are easily seen to satisfy $\left(A^{T}\right)_{\eta \kappa}=\left(A_{\kappa \eta}\right)^{T}$, generalizing the defining property of the transpose, $\left(A^{T}\right)_{i j}=A_{j i}$. When $\eta=\kappa$, then $A_{\eta \kappa}$ is a principal submatrix of $A$. When $\eta \neq \kappa$, we refer to $A_{\eta \kappa}$ and $A_{\kappa \eta}$ as a dual pair of submatrices of $A$, and their determinants as dual minors. Reversal pairs arise naturally in the investigation of dual minors as the following lemma shows.

Lemma 6.2 (Dual minors of $T$-palindromic polynomials). If $P(\lambda)$ is an $n \times n$ $T$-palindromic polynomial of grade $j$, then any pair of dual minors of $P(\lambda)$ of size $\ell \leq n$ forms a jl-reversal pair.

Proof. For any index sets $\eta$ and $\kappa$ of size $\ell$, we wish to show that the dual minors $\operatorname{det} P_{\eta \kappa}(\lambda)$ and $\operatorname{det} P_{\kappa \eta}(\lambda)$ form a $j \ell$-reversal pair. From $P(\lambda)$ being $T$-palindromic of grade $j$, we immediately have

$$
\left[\lambda^{j} P^{T}(1 / \lambda)\right]_{\eta \kappa}= \pm P_{\eta \kappa}(\lambda) .
$$

The basic relation $\left(A^{T}\right)_{\eta \kappa}=\left(A_{\kappa \eta}\right)^{T}$ between dual submatrices then implies that

$$
\lambda^{j}\left[P_{\kappa \eta}(1 / \lambda)\right]^{T}= \pm P_{\eta \kappa}(\lambda) .
$$

Taking determinants we see that

$$
\lambda^{j \ell} \operatorname{det}\left(P_{\kappa \eta}(1 / \lambda)\right)= \pm \operatorname{det} P_{\eta \kappa}(\lambda) .
$$

Since the degree of any $\ell \times \ell$ minor of $P(\lambda)$ is at most $j \ell$, equation (6.1) is the same as

$$
\left(\operatorname{rev}_{j \ell}\left(\operatorname{det} P_{\kappa \eta}\right)\right)(\lambda)= \pm \operatorname{det} P_{\eta \kappa}(\lambda),
$$

showing that $\operatorname{det} P_{\kappa \eta}(\lambda)$ and $\operatorname{det} P_{\eta \kappa}(\lambda)$ form a $j \ell$-reversal pair.

To obtain the invariant polynomials in the Smith form, we need the GCDs of minors of the matrix polynomial. The following lemma, whose straightforward proof can be found in [22], will be helpful in showing that if we start with a $T$-palindromic matrix polynomial, then the GCD's of interest are also palindromic.

Lemma 6.3 (Subset collapsing lemma for GCD's). Suppose $S$ is a finite set of polynomials, and $S=S_{1} \cup S_{2}$ for some nonempty subsets $S_{1}$ and $S_{2}$ (not necessarily disjoint). Then $\operatorname{gcd}(S)=\operatorname{gcd}\left(S_{1} \cup\left\{\operatorname{gcd}\left(S_{2}\right)\right\}\right)$.

TheOrem 6.4 (GCD of $\ell \times \ell$ minors of $T$-palindromic polynomials). If $P$ is an $n \times n$ T-palindromic matrix polynomial of grade $k$, then for each $\ell=1, \ldots, n$, the $G C D$ of the set of all $\ell \times \ell$ minors of $P$ is palindromic. 
Proof. The set of all $\ell \times \ell$ minors of $P(\lambda)$ may be grouped into the principal minors and the dual minor pairs. By Proposition 6.1, any principal $\ell \times \ell$ minor of $P(\lambda)$ is palindromic (specifically, $k \ell$-palindromic). By Lemma 6.2, each $\ell \times \ell$ dual minor pair is a reversal pair (in particular, a $k \ell$-reversal pair), and by Proposition 5.15, the GCD of any reversal pair is palindromic. Thus by the "subset collapsing" Lemma 6.3, the GCD of the set of all $\ell \times \ell$ minors of $P(\lambda)$ is the same as the GCD of a set consisting entirely of palindromic polynomials. By Proposition 5.10, this GCD must be palindromic.

7. $\mathbf{P}-S_{\text {Smith }}{ }^{1}$ form. The results established so far immediately show that palindromicity of a matrix polynomial is inherited by its invariant polynomials: by Theorem 2.2 each invariant polynomial is a ratio of GCD's, by Theorem 6.4 each of these GCD's is palindromic, and by Lemma 5.3(c) each of these ratios is palindromic. The next proposition formally states this result.

Proposition 7.1. Suppose that $D(\lambda)=\operatorname{diag}\left(d_{1}(\lambda), d_{2}(\lambda), \ldots, d_{n}(\lambda)\right)$ is the Smith form of a T-palindromic matrix polynomial $P(\lambda)$. Then each $d_{i}(\lambda)$ is palindromic.

Note that while each diagonal entry of $P$ has the same grade of palindromicity as $P$ (Example 5.2), the diagonal entries of $D$ will likely have varying grades of palindromicity.

For the rest of this section, we assume that the underlying field $\mathbb{F}$ has char $\mathbb{F} \neq 2$; the case of char $\mathbb{F}=2$ will be discussed in Appendix A. It will be useful to know how the grade and the palindromic type are altered under some simple transformations.

LEMMA 7.2. If $P$ is a T-palindromic matrix polynomial of grade $j$ over a field $\mathbb{F}$ with char $\mathbb{F} \neq 2$, then

(a) $R(\lambda):=(\lambda-1) P(\lambda)$ is T-palindromic of grade $(j+1)$ and of opposite palindromic type, that is, $\varepsilon(R)=-\varepsilon(P)$.

(b) $Q(\lambda):=P(-\lambda)$ is T-palindromic of the same grade $j$, and palindromic type $\varepsilon(Q)=(-1)^{j} \varepsilon(P)$, i.e., the palindromic type switches when the grade is odd.

Proof. (a): We have

$$
\left(\operatorname{rev}_{j+1} R\right)(\lambda)=\lambda^{j+1} R(1 / \lambda)=\lambda \lambda^{j}\left(\frac{1}{\lambda}-1\right) P(1 / \lambda)=-(\lambda-1)\left(\operatorname{rev}_{j} P\right)(\lambda) .
$$

Since $P$ is $T$-palindromic, $\left(\operatorname{rev}_{j} P\right)(\lambda)=\varepsilon(P) P^{T}(\lambda)$. Hence

$$
\left(\operatorname{rev}_{j+1} R\right)(\lambda)=-\varepsilon(P)(\lambda-1) P^{T}(\lambda)=-\varepsilon(P) R^{T}(\lambda)
$$

\footnotetext{
${ }^{1}$ By contrast with [27], here the $\mathrm{P}$ is not silent.
} 
showing $R$ is $T$-palindromic of grade $(j+1)$ and palindromic type $\varepsilon(R)=-\varepsilon(P)$.

(b): We have

$\left(\operatorname{rev}_{j} Q\right)(\lambda)=\lambda^{j} Q(1 / \lambda)=\lambda^{j} P(-1 / \lambda)=(-1)^{j}(-\lambda)^{j} P(-1 / \lambda)=(-1)^{j}\left(\operatorname{rev}_{j} P\right)(-\lambda)$.

Since $P$ is $T$-palindromic, $\left(\operatorname{rev}_{j} P\right)(-\lambda)=\varepsilon(P) P^{T}(-\lambda)$, and so

$$
\left(\operatorname{rev}_{j} Q\right)(\lambda)=(-1)^{j} \varepsilon(P) Q^{T}(\lambda),
$$

yielding the desired result.

The relation between the elementary divisors of $P, Q$ and $R$ as introduced in Lemma 7.2 is straightforward, and can be conveniently stated using the notion of partial multiplicity sequence from Definition 2.3.

Lemma 7.3. Let $P$ be an arbitrary matrix polynomial over a field $\mathbb{F}$ with char $\mathbb{F} \neq 2$, and let the partial multiplicity sequence of $P$ at $\lambda_{0} \in \mathbb{F}$ be $\left(\alpha_{1}, \alpha_{2}, \ldots, \alpha_{\ell}\right)$. Define $R(\lambda):=\left(\lambda-\lambda_{0}\right) P(\lambda)$ and $Q(\lambda):=P(-\lambda)$. Then

(a) the partial multiplicity sequence of $R$ at $\lambda_{0}$ is $\left(\alpha_{1}+1, \alpha_{2}+1, \ldots, \alpha_{\ell}+1\right)$, and

(b) the partial multiplicity sequence of $Q$ at $-\lambda_{0}$ is $\left(\alpha_{1}, \alpha_{2}, \ldots, \alpha_{\ell}\right)$.

Proof. If $D(\lambda)=E(\lambda) P(\lambda) F(\lambda)$ is the Smith form of $P$, where $E, F$ are unimodular, then $\left(\lambda-\lambda_{0}\right) D(\lambda)=E(\lambda) R(\lambda) F(\lambda)$ is the Smith form of $R$, yielding (a). Next, the identity $D(-\lambda)=E(-\lambda) Q(\lambda) F(-\lambda)$ shows that $D(-\lambda)$ is almost the Smith form of $Q$; it only remains to make all its diagonal entries monic. But this is easily done by multiplying by an appropriate constant matrix. Since any factor $\left(\lambda-\lambda_{0}\right)$ in $D(\lambda)$ effectively becomes $\left(\lambda+\lambda_{0}\right)$ in $D(-\lambda)$, result (b) now follows.

Definition 7.4 (Pairing property $\Phi$ ). Let $\alpha_{1} \leq \alpha_{2} \leq \cdots \leq \alpha_{\ell}$ be any ordered sequence. Then a subsequence

$$
\alpha_{h_{1}} \leq \alpha_{h_{2}} \leq \cdots \leq \alpha_{h_{\nu}} \quad \text { with } \quad h_{1}<h_{2}<\cdots<h_{\nu}
$$

has the pairing property $\Phi$ if $\nu=2 m>0$ is even, and the following conditions hold:

(a) Adjacency pairing of terms:

$$
h_{2}=h_{1}+1, \quad h_{4}=h_{3}+1, \quad \ldots, \quad h_{2 m}=h_{2 m-1}+1 .
$$

(b) Equality pairing of adjacent terms:

$$
\alpha_{h_{2}}=\alpha_{h_{1}}, \quad \alpha_{h_{4}}=\alpha_{h_{3}}, \quad \ldots, \quad \alpha_{h_{2 m}}=\alpha_{h_{2 m-1}} .
$$

Example 7.5. In the sequence $(0,0, \mathbf{1}, \mathbf{1}, 2,4,4, \mathbf{5}, \mathbf{5}, \mathbf{5}, \mathbf{5}, 8, \mathbf{9}, \mathbf{9})$, the subsequence of all odd integers (indicated in bold face) has the pairing property $\Phi$. 
We are now ready to state one of the main results of this paper — the influence of the $T$-palindromicity of $P$ on its invariant polynomials. Note that when $\operatorname{deg} P(\lambda)=0$, the nonzero diagonal entries of its Smith form are all just 1.

ThEOREM 7.6 (P-Smith form). Let $P(\lambda)$ be a nonzero $n \times n$ T-palindromic matrix polynomial of grade $k$ over an arbitrary field $\mathbb{F}$ with char $\mathbb{F} \neq 2$. Suppose that the Smith form of $P(\lambda)$ is expressed as

$$
D(\lambda)=\operatorname{diag}\left((\lambda-1)^{\alpha_{1}}(\lambda+1)^{\beta_{1}} p_{1}(\lambda), \ldots,(\lambda-1)^{\alpha_{\ell}}(\lambda+1)^{\beta_{\ell}} p_{\ell}(\lambda), 0, \ldots, 0\right)
$$

where $\ell \leq n, 0 \leq \alpha_{1} \leq \cdots \leq \alpha_{\ell}$ and $0 \leq \beta_{1} \leq \cdots \leq \beta_{\ell}$ are nonnegative integers, $p_{j}(\lambda)$ is monic with $p_{j}(1) \neq 0$ and $p_{j}(-1) \neq 0$ for $j=1, \ldots, \ell$, and $p_{j}(\lambda) \mid p_{j+1}(\lambda)$ for $j=1, \ldots, \ell-1$. Then

(1) $p_{j}(\lambda)$ is palindromic of type $\varepsilon\left(p_{j}\right)=+1$, for $j=1, \ldots, \ell$, and

(2) the pairing property $\Phi$ holds for the following partial multiplicity subsequences of $P$ at $\lambda_{0}=1$ and $\lambda_{0}=-1$ :

\begin{tabular}{|c|c|c|c|}
\hline \multicolumn{2}{|c|}{$P(\lambda)$} & \multicolumn{2}{c|}{ Partial mult. subsequences of $P$ with property $\Phi$} \\
\hline Type $\varepsilon(P)$ & Grade $k$ & At $\lambda_{0}=1$ & $A t \lambda_{0}=-1$ \\
\hline \hline \multirow{2}{*}{+1} & even & (a) All odd $\alpha_{i}$ 's & (e) All odd $\beta_{j}$ 's \\
\cline { 2 - 4 } & odd & (b) All odd $\alpha_{i}$ 's & (f) All even $\beta_{j}$ 's \\
\hline \multirow{2}{*}{-1} & even & (c) All even $\alpha_{i}$ 's & (g) All even $\beta_{j}$ 's \\
\cline { 2 - 4 } & odd & (d) All even $\alpha_{i}$ 's & (h) All odd $\beta_{j}$ 's \\
\hline
\end{tabular}

We emphasize that "all even" in $(c),(d),(f),(g)$, includes any and all occurrences of the exponent 0 .

Proof. (1): That each $p_{j}$ is palindromic follows from Proposition 7.1 and Lemma 5.3(c). If $p_{j}$ was anti-palindromic, then $(\lambda-1)$ would be a factor by Lemma 5.6(b), contradicting $p_{j}(1) \neq 0$. Hence, $\varepsilon\left(p_{j}\right)=1$ for $j=1, \ldots, \ell$.

(2): Suppose we have established (a) and (b) for all palindromic polynomials of type +1 . Then $(\mathrm{c})-(\mathrm{h})$ follow using previous lemmas. In particular, if $P(\lambda)$ is anti-palindromic, then Lemma 7.2(a) says that the palindromic polynomial $R(\lambda):=$ $(\lambda-1) P(\lambda)$ has opposite type, and grade of opposite parity. Applying (a), (b) to $R$ and using Lemma 7.3(a) yield (d) and (c), respectively, for $P$. Next, setting $Q(\lambda):=$ $P(-\lambda)$, we deduce from the second parts of Lemmas 7.2 and 7.3 that (a) implies (e), (b) implies (h), (c) implies (g), and finally, (d) implies (f).

Thus, it only remains to prove (a) and (b). So let $P(\lambda)$ be a non-constant palindromic polynomial with $\varepsilon(P)=1$. Making no assumption on the parity of its grade 
$k$, we show that the subsequence

$$
\alpha_{h_{1}} \leq \alpha_{h_{2}} \leq \cdots \leq \alpha_{h_{\nu}}
$$

of all odd terms in the partial multiplicity sequence of $P$ at $\lambda_{0}=1$ has the pairing property $\Phi$.

The strategy is similar to the one used in the proof of [22, Theorem 3.10]. We first show that if $\alpha_{1}$ is odd, then $\alpha_{2}=\alpha_{1}$. Next, we use the results from Section 2.2 on compound matrices to push the pairing properties down the diagonal of $D(\lambda)$.

Step 1: We show that if $\alpha_{1}$ is odd, then $n \geq 2, \ell \geq 2$, and $\alpha_{2}=\alpha_{1}$.

Since $\alpha_{1}$ is odd, $d_{1}(\lambda)$ is a nonzero anti-palindromic polynomial. From Theorem 2.2, we know that $d_{1}(\lambda)$ is the GCD of all the entries of $P(\lambda)$. Now the diagonal entries of any $T$-palindromic matrix polynomial $P(\lambda)$ are scalar palindromic polynomials of the same type as $P(\lambda)$, so in this case they are palindromic of type +1 . Thus by Lemma 5.7(b), each diagonal entry must contain an even number of $(\lambda-1)$ factors. We can therefore write $P(\lambda)_{j j}=(\lambda-1)^{\alpha_{1}+1} r_{j}(\lambda)$, where each $r_{j}(\lambda)$ is palindromic of type +1 , by Lemma 5.3(c). (Note that we allow $r_{j}$ to be zero.) But $d_{1}(\lambda)=$ $(\lambda-1)^{\alpha_{1}}(\lambda+1)^{\beta_{1}} p_{1}(\lambda)$ being the GCD of all the entries of $P(\lambda)$ implies that there must be some off-diagonal entry $P(\lambda)_{i j}$ with $i>j$ of the form $(\lambda-1)^{\alpha_{1}} s(\lambda)$ with $s(1) \neq 0$, and consequently that $n \geq 2$. Let $\eta=\{i, j\}$ and consider the corresponding $2 \times 2$ principal submatrix

$$
P(\lambda)_{\eta \eta}=\left[\begin{array}{cc}
(\lambda-1)^{\alpha_{1}+1} r_{i}(\lambda) & \operatorname{rev}_{k} P(\lambda)_{i j} \\
P(\lambda)_{i j} & (\lambda-1)^{\alpha_{1}+1} r_{j}(\lambda)
\end{array}\right]
$$

Now with $P(\lambda)_{i j}=(\lambda-1)^{\alpha_{1}} s(\lambda)$, we have

$$
\operatorname{rev}_{k} P(\lambda)_{i j}=\lambda^{k}(1 / \lambda-1)^{\alpha_{1}} s(1 / \lambda)=(-1)^{\alpha_{1}} \lambda^{k-\alpha_{1}}(\lambda-1)^{\alpha_{1}} s(1 / \lambda),
$$

so that

$$
\operatorname{det} P(\lambda)_{\eta \eta}=(\lambda-1)^{2 \alpha_{1}}[\underbrace{(\lambda-1)^{2} r_{i}(\lambda) r_{j}(\lambda)+(-1)^{\alpha_{1}+1} \lambda^{k-\alpha_{1}} s(\lambda) s(1 / \lambda)}_{=: w(\lambda)}] .
$$

Now observe that $w(\lambda)$ is a nonzero polynomial, as $s(1) \neq 0$ implies that $w(1) \neq 0$. Thus det $P(\lambda)_{\eta \eta}$ is a nonzero polynomial, and hence the GCD $g(\lambda)$ of all the $2 \times 2$ minors is nonzero, so $\ell \geq 2$. Furthermore, from (7.2) we see that $g(\lambda)$ contains a power of $(\lambda-1)$ no higher than $2 \alpha_{1}$. Now recall from Theorem 2.2 that

$$
g(\lambda)=d_{1}(\lambda) d_{2}(\lambda)=(\lambda-1)^{\alpha_{1}+\alpha_{2}}(\lambda+1)^{\beta_{1}+\beta_{2}} p_{1}(\lambda) p_{2}(\lambda) .
$$

Since $p(1) \neq 0$ and $p_{2}(1) \neq 0$, we must have $\alpha_{1}+\alpha_{2} \leq 2 \alpha_{1}$. By hypothesis, $\alpha_{1} \leq \alpha_{2}$, and so $\alpha_{2}=\alpha_{1}$ as desired. 
Step 2: Push forward down the diagonal.

Assume the contrary, i.e., the positions $h_{1}<h_{2}<\cdots<h_{\nu}$ where the odd exponents of the factor $(\lambda-1)$ occur do not satisfy the pairing property $\Phi$. Let $r:=h_{2 j-1}$ be the first position where $\Phi$ fails. Then one of the following must occur:

- $\nu=2 j-1$ so that $h_{2 j}$ does not exist, or

- $h_{2 j}$ exists, but adjacency-pairing fails, i.e., $h_{2 j} \neq h_{2 j-1}+1$, or

- $h_{2 j}$ exists and $h_{2 j-1}, h_{2 j}$ are adjacent, but $\alpha_{h_{2 j}} \neq \alpha_{h_{2 j-1}}$.

First observe that if $r=n$, then $\operatorname{det} D(\lambda)$, and hence also $\operatorname{det} P(\lambda)$, have an odd number of $(\lambda-1)$ factors. This contradicts Lemma $5.7(\mathrm{~b})$, since $\operatorname{det} P(\lambda)$ is palindromic of type +1 , by Proposition 6.1. Hence, $r<n$.

Now consider the $r$ th compound matrix of $P(\lambda)$. By Theorem 2.6, the first two invariant polynomials of $\mathcal{C}_{r}[P(\lambda)]$ are

$$
c_{1}(\lambda)=d_{1}(\lambda) \cdots d_{r-1}(\lambda) d_{r}(\lambda) \quad \text { and } \quad c_{2}(\lambda)=d_{1}(\lambda) \cdots d_{r-1}(\lambda) d_{r+1}(\lambda) .
$$

Since $d_{r}(\lambda)$ is the $(2 j-1)$ st nonzero invariant polynomial $P(\lambda)$ with an odd number of $(\lambda-1)$ factors, we see that $c_{1}(\lambda)$ must also be nonzero and have an odd number of $(\lambda-1)$ factors. Now $\mathcal{C}_{r}[P(\lambda)]$ is $T$-palindromic of type +1 , by Theorem 4.6. Since its first invariant polynomial $c_{1}(\lambda)$ has an odd number of $(\lambda-1)$ factors, by Step 1 the second invariant polynomial $c_{2}(\lambda)$ must have exactly the same number of $(\lambda-1)$ factors as $c_{1}(\lambda)$. Consequently $d_{r+1}(\lambda)$ must be nonzero with the same (odd) number of $(\lambda-1)$ factors as $d_{r}(\lambda)$, so that the ratio $d_{r+1}(\lambda) / d_{r}(\lambda)$ is not divisible by $(\lambda-1)$. Thus $h_{2 j}=r+1$ exists, is adjacent to $h_{2 j-1}$, and $\alpha_{h_{2 j-1}}=\alpha_{h_{2 j}}$, contradicting the assumption that $\Phi$ fails at $h_{2 j-1}$. The proof is now complete.

In contrast to the situation for $T$-alternating polynomials [22, Theorems 3.10 , 3.11], the conditions in Theorem 7.6 are necessary but not sufficient for a diagonal matrix to be the Smith form of some $T$-palindromic matrix polynomial. This is illustrated by the following example.

Example 7.7. Consider the matrix $D(\lambda)=\operatorname{diag}\left(1, \lambda^{2}+1,\left(\lambda^{2}+1\right)\left(\lambda^{4}+\lambda^{2}+1\right)\right)$. Its diagonal entries are monic, palindromic of type +1 , and satisfy the divisibility requirements to be a Smith form. Since \pm 1 are not eigenvalues, $D(\lambda)$ satisfies all the conditions of Theorem 7.6. Observe, however, that each diagonal entry has a different grade of palindromicity $(0,2$, and 6 , respectively), so $D$ itself cannot be $T$-palindromic (Example 5.2). But neither can $D$ be the Smith form of a $3 \times 3 T$-palindromic polynomial of any grade $k$. If it were, then by Proposition 6.1 , $\operatorname{det} D(\lambda)$ would have to be $3 k$-palindromic. However, $\operatorname{det} D(\lambda)$ is 8 -palindromic, by Lemma $5.3(\mathrm{~b})$.

Thus we see that a condition of a more "global" nature, involving both the grade of palindromicity and the size of the matrix is required for sufficiency. Determining conditions that are both necessary and sufficient for a given diagonal matrix to be 
the Smith form of some $T$-palindromic matrix polynomial remains an open problem.

REMARK 7.8. It is interesting to note the presence of some non-trivial facts hiding inside the P-Smith theorem, disguised in the seemingly trivial form of partial multiplicity sequences consisting of all zeroes. For example, suppose $P(\lambda)$ is $T$-antipalindromic (i.e., $\varepsilon(P)=-1$ ) and $\lambda_{0}=1$ is not an eigenvalue, so that the partial multiplicity sequence of $P$ at $\lambda_{0}=1$ is all zeroes. Then we are in case (c) or (d) of the table in Theorem 7.6, and can conclude that $\ell=\operatorname{rank} P(\lambda)$ must be even! If in addition $\operatorname{deg} P$ is zero (more apparent triviality), then a $T$-anti-palindromic $P(\lambda)$ is just a skew-symmetric matrix (with no eigenvalues as a matrix polynomial at all), and so we unexpectedly recover, as a very special case of Theorem 7.6, the well-known but non-trivial result that any skew-symmetric matrix over a field $\mathbb{F}$ with char $\mathbb{F} \neq 2$ has even rank.

7.1. The $*$-palindromic case. Theorem 7.6 naturally leads one to ask what the necessary conditions on the Smith form of $*$-palindromic polynomials might be. Because all regular $*$-palindromic polynomials have palindromic linearizations [21], we expect that there will be fewer conditions on the Smith form, and a simpler story to tell, as we now see.

A matrix polynomial over the field of complex numbers is $*$-palindromic when there exists an integer $j \geq \operatorname{deg}(P)$ such that $\operatorname{rev}_{j} P(\lambda)=P^{*}(\lambda)$. Note that it is sufficient to only consider $*$-palindromic matrix polynomials of type +1 , because any $*$-palindromic matrix polynomial of type -1 , i.e., one satisfying $\operatorname{rev}_{j} P(\lambda)=-P^{*}(\lambda)$, can be transformed into a $*$-palindromic matrix polynomial of type +1 by multiplying with the scalar $i$.

In the scalar case a polynomial $p$ is $*$-palindromic if $\operatorname{rev}_{j} p(x)=\bar{p}(x)$, and we say $p$ is conjugate palindromic of grade $j$.

Several results established for scalar palindromic polynomials also hold for conjugate palindromic polynomials $p(x)$, after some straightforward modifications. For example, every irreducible factor $d(x) \neq x$ that is not a scalar multiple of a conjugate palindromic comes paired with a distinct irreducible factor of the form rev $\bar{d}(x)$. Then $d(x) \cdot \operatorname{rev} \bar{d}(x)$ is a conjugate palindromic factor of $p(x)$ of grade equal to twice the degree of $d$ (the analog of Lemma 5.5).

There is a significant difference, however, when one considers conjugate palindromic polynomials of degree one: $d(x)=\alpha x+\bar{\alpha}$ is conjugate palindromic for any $\alpha \in \mathbb{C}$, so the polynomials $x-1$ and $x+1$ no longer play a distinguished role. Consequently, the factorization of a conjugate palindromic scalar polynomial into a product of irreducibles takes on a different look.

Theorem 7.9 (Irreducible $*$-palindromic factorization). A polynomial $p(x) \in$ 
$\mathbb{C}[x]$ is conjugate palindromic if and only if it admits an irreducible factorization of the form

$$
p(x)=c \cdot x^{k} \cdot \prod_{i=1}^{\mu}\left(a_{i} x+\bar{a}_{i}\right)^{m_{i}} \cdot \prod_{j=1}^{\nu}\left(\left(x+b_{j}\right)\left(\bar{b}_{j} x+1\right)\right)^{n_{j}},
$$

where $c \in \mathbb{R}, a_{i}, b_{j} \in \mathbb{C},\left|a_{i}\right|=1,\left|b_{j}\right| \neq 1, k, m_{1}, \ldots, m_{\mu}, n_{1}, \ldots, n_{\nu} \in \mathbb{N}$.

A $j$-conjugate-reversal pair of scalar polynomials $p$ and $q$ is a pair of nonzero polynomials that satisfy $\operatorname{rev}_{j} p=\bar{q}$ for some $j \geq \max \{\operatorname{deg} p, \operatorname{deg} q\}$. One can show that the GCD of such a reversal pair is conjugate palindromic up to a scalar multiple (since the GCD is by definition monic). It follows that for each $\ell=1, \ldots, n$, the GCD of the set of all $\ell \times \ell$ minors of an $n \times n *$-palindromic matrix polynomial is conjugate palindromic up to a scalar multiple (the analog of Theorem 6.4). Then just as before, one can show that $*$-palindromicity of a matrix polynomial is inherited by its invariant polynomials, modulo multiplication by constants. The proofs are straightforward modifications of the analogs for the $T$-palindromic case, and so are omitted.

TheOREm 7.10 (Smith form for *-palindromic polynomials). Let $P(\lambda)$ be a nonzero $n \times n$ matrix polynomial over $\mathbb{F}=\mathbb{C}$ that is $*$-palindromic. Suppose the Smith form of $P(\lambda)$ is given by $D(\lambda)=\operatorname{diag}\left(d_{1}(\lambda), d_{2}(\lambda), \ldots, d_{n}(\lambda)\right)$. Then there exist nonzero constants $c_{i} \in \mathbb{C}$ such that each $c_{i} d_{i}(\lambda)$ is conjugate palindromic, that $i s,\left(\operatorname{rev}\left(c_{i} d_{i}\right)\right)(\lambda)=\overline{c_{i} d_{i}}(\lambda)$.

In contrast to the $T$-palindromic case, there are no additional restrictions on the elementary divisors corresponding to the eigenvalues \pm 1 , or, more generally, corresponding to eigenvalues on the unit circle. Indeed, suppose that $c_{1} d_{1}(\lambda), \ldots, c_{\ell} d_{\ell}(\lambda)$ are nonzero conjugate palindromic scalar polynomials, where $c_{i} \in \mathbb{C}$ are nonzero constants, and $d_{i}$ are monic polynomials with the divisibility property $d_{i} \mid d_{i+1}$ for $i=1, \ldots, \ell-1$. If there is a $*$-palindromic matrix polynomial with these $d_{i}$ as its invariant polynomials, then it follows from Theorem 7.9 that elementary divisors associated with any finite nonzero eigenvalue $\lambda_{0}$ not lying on the unit circle occur in pairs $\left(\lambda-\lambda_{0}\right)^{m},\left(\lambda-1 / \bar{\lambda}_{0}\right)^{m}$, whereas there is no enforced pairing for elementary divisors associated with eigenvalues on the unit circle. After pairing up each elementary divisor $\lambda^{k}$ with an infinite elementary divisor of the same degree, one can immediately construct a $*$-palindromic pencil having exactly this collection of elementary divisors by using [25, Theorem 2]. In particular, the invariant polynomials of such a pencil will be $d_{1}(\lambda), \ldots, d_{\ell}(\lambda)$, except possibly for additional leading ones.

8. Jordan structure. Now that we know the structure of the Smith forms of $T$-palindromic matrix polynomials, we can interpret these results in terms of their Jordan structure. In earlier work [21, Theorem 2.2] it was shown that the eigenvalues 
of a regular complex $T$-palindromic polynomial $P$ occur in $(\lambda, 1 / \lambda)$ pairs with the same algebraic, geometric and partial multiplicities. This earlier proof cannot be adapted to the singular case, as it relies on the strict equivalence of the first and second companion forms, which was recently shown to completely break down whenever $P$ is singular [3, Corollary 5.11], [4, Corollary 6.2]. By contrast, the proof of Theorem 7.6 establishing the structured Smith form makes no assumption on the regularity of $P$. Consequently we can extend the reciprocal pairing symmetry of the elementary divisors to all $T$-palindromic polynomials, singular as well as regular.

COROLlary 8.1 (Reciprocal pairing). Let $\lambda_{0}$ be an eigenvalue of a T-palindromic polynomial $P(\lambda)$, over an arbitrary field $\mathbb{F}$ with char $\mathbb{F} \neq 2$. Then $1 / \lambda_{0}$ is also an eigenvalue of $P(\lambda)$. Furthermore, the partial multiplicity sequences of $P$ at $\lambda_{0}$ and $1 / \lambda_{0}$ are the same, and hence their algebraic and geometric multiplicities agree. (Here, we interpret 0 and $\infty$ as reciprocal pairs.)

Proof. If $\lambda_{0}= \pm 1$, then there is nothing to prove. Otherwise, let $D(\lambda)$ as in (7.1) be the Smith form of $P$. By Theorem 7.6, each $p_{i}(\lambda)$ is palindromic, and hence has a factorization of the form (5.1). This means that whenever $\left(\lambda-\lambda_{0}\right)^{\alpha}$ with $\lambda_{0} \neq 0$ is a factor of $p_{i}$, then so is $\left(\lambda-1 / \lambda_{0}\right)^{\alpha}$. Hence $1 / \lambda_{0}$ is also an eigenvalue of $P$ with the same partial multiplicity sequence as $\lambda_{0}$, proving the result for nonzero finite eigenvalues. Since $P$ is $T$-palindromic, $\operatorname{rev}_{k} P= \pm P^{T}$, so $\operatorname{rev}_{k} P$ and $P$ have the same Smith form $D$, yielding the desired pairing for zero and infinite eigenvalues as well.

While Corollary 8.1 adds no information about the special elementary divisors $(\lambda \pm 1)^{\alpha}$, Theorem 7.6 specifies conditions when the partial multiplicity sequences at $\lambda_{0}= \pm 1$ have subsequences with the pairing property $\Phi$. As an immediate consequence, we have the following situations when the elementary divisors $(\lambda \pm 1)^{\alpha}$ necessarily have even multiplicity.

Corollary 8.2 (Even multiplicity of $\left.(\lambda \pm 1)^{\alpha}\right)$. Let $P$ be a T-palindromic matrix polynomial of degree at least 1 and grade $k$, over an arbitrary field $\mathbb{F}$ with char $\mathbb{F} \neq 2$. Let $\left(\lambda-\lambda_{0}\right)^{\alpha}$ be an elementary divisor of $P$, for some positive integer $\alpha$. For $\lambda_{0}= \pm 1$, the following table gives conditions when $\left(\lambda-\lambda_{0}\right)^{\alpha}$ must have even multiplicity.

\begin{tabular}{|c|c|c|c|}
\hline \multicolumn{2}{|c|}{$P(\lambda)$} & \multicolumn{2}{c|}{$\begin{array}{c}\text { Elementary divisors }\left(\lambda-\lambda_{0}\right)^{\alpha} \\
\text { with guaranteed even multiplicity }\end{array}$} \\
\hline Type $\varepsilon(P)$ & Grade $k$ & For $\lambda_{0}=1$ & For $\lambda_{0}=-1$ \\
\hline \hline \multirow{2}{*}{+1} & even & odd $\alpha$ & odd $\alpha$ \\
\cline { 2 - 4 } & odd & odd $\alpha$ & even $\alpha$ \\
\hline \multirow{2}{*}{-1} & even & even $\alpha$ & even $\alpha$ \\
\cline { 2 - 4 } & odd & even $\alpha$ & odd $\alpha$ \\
\hline
\end{tabular}


With $k=1$ as a special case, we can now obtain results on the Jordan structure of $T$-palindromic matrix pencils. For eigenvalues $\lambda_{0} \neq \pm 1$, the reciprocal pairing detailed in Corollary 8.1 directly applies. Using Corollary 8.2 with $k=1$ yields Corollary 8.3, telling us when $(\lambda \pm 1)^{\alpha}$ must have even multiplicity. Our results over arbitrary fields give independent proofs of the elementary divisor pairing results presented in $[25,26]$ for $T$-palindromic pencils over the fields $\mathbb{F}=\mathbb{C}$ and $\mathbb{F}=\mathbb{R}$; see also the related results in $[14]$.

Corollary 8.3 (Even multiplicity of $(\lambda \pm 1)^{\alpha}$ for pencils). Let $L(\lambda)=\lambda Z \pm Z^{T}$ be a non-zero $T$-palindromic pencil over an arbitrary field $\mathbb{F}$ with char $\mathbb{F} \neq 2$. Let $\left(\lambda-\lambda_{0}\right)^{\alpha}$ be an elementary divisor of $L$, for some positive integer $\alpha$. When $\lambda_{0}= \pm 1$, the following table gives conditions when $\left(\lambda-\lambda_{0}\right)^{\alpha}$ must have even multiplicity.

\begin{tabular}{|c|c|c|}
\hline \multirow{2}{*}{$L(\lambda)$} & \multicolumn{2}{|c|}{$\begin{array}{c}\text { Elementary divisors }\left(\lambda-\lambda_{0}\right)^{\alpha} \\
\text { with guaranteed even multiplicity }\end{array}$} \\
\cline { 2 - 3 } & $\lambda_{0}=1$ & $\lambda_{0}=-1$ \\
\hline \hline$\lambda Z+Z^{T}$ & odd $\alpha$ & even $\alpha$ \\
\hline$\lambda Z-Z^{T}$ & even $\alpha$ & odd $\alpha$ \\
\hline
\end{tabular}

9. Structured linearizations. We now use Theorem 7.6 on the P-Smith form and the corollaries in Section 8 on the elementary divisor structure of $T$-palindromic matrix polynomials to address the question that was the original motivation for this paper — when does a $T$-palindromic polynomial have a $T$-palindromic linearization? A necessary condition for the existence of such a structured linearization is immediately clear.

ThEOREM 9.1 (Compatibility of Jordan structures at $\lambda_{0}= \pm 1$ ). In order for a T-palindromic polynomial $P$ to have a T-palindromic linearization, the elementary divisors of $P$ at $\lambda_{0}= \pm 1$ must be compatible with those of $T$-palindromic pencils, as described in Corollary 8.3.

One striking feature clearly displayed in Corollary 8.2 is that the elementary divisor structure of a $T$-palindromic $P$ depends only on the palindromic type of $P$ and the parity of the grade of $P$. As a consequence, for the structured linearization question we see a fundamental dichotomy between the behavior of even grade and odd grade palindromic polynomials. It is possible for an even grade palindromic polynomial to have an elementary divisor structure that is incompatible with every palindromic pencil, of either +1 or -1 type, and thus to have no palindromic linearization of any kind. This is illustrated in the following example. 
Example 9.2. Consider the type +1 quadratic $T$-palindromic polynomial

$$
Q(\lambda)=\left[\begin{array}{cc}
\lambda^{2}+1 & 2 \lambda \\
2 \lambda & \lambda^{2}+1
\end{array}\right]
$$

Using the GCD characterization of invariant polynomials described in Theorem 2.2, it is not hard to see that the Smith form of $Q(\lambda)$ is $\operatorname{diag}\left(1,(\lambda-1)^{2}(\lambda+1)^{2}\right)$. The elementary divisors are $(\lambda-1)^{2}$ and $(\lambda+1)^{2}$, both occurring once, so both have odd multiplicity. By Corollary 8.3, this elementary divisor structure cannot occur in any palindromic pencil, so $Q(\lambda)$ has no palindromic linearization. The same type of argument also explains why the palindromic polynomial $P(\lambda)=\lambda^{2}-1$ from Example 1.1 cannot have a palindromic linearization either.

On the other hand, the kind of incompatibility seen in Example 9.2 can never occur for an odd grade palindromic polynomial. In fact, a comparison of Corollaries 8.2 and 8.3 strongly suggests that every palindromic polynomial of odd grade should have a palindromic linearization of the same palindromic type. We will see in Section 9.1 that this is indeed the case.

The even grade case is more difficult, and requires considerably more work to settle. We briefly discuss some of the issues in Section 9.2, but the complete resolution of the problem is currently under investigation.

9.1. The odd grade case. We now describe a simple procedure to construct a strong $T$-palindromic linearization for any $T$-palindromic matrix polynomial $P(\lambda)$ of odd grade. The construction works equally well for regular and singular $P(\lambda)$ of odd grade $k$, over an arbitrary field $\mathbb{F}$ with char $\mathbb{F} \neq 2$. It exploits a family of blocktridiagonal $k n \times k n$ pencils $\mathcal{S}_{P}(\lambda)$ that are built directly from the matrix coefficients of $P(\lambda)$. These pencils were introduced and analyzed in [22], extending and simplifying results in [1]. For example, to $P(\lambda)=\lambda^{3} A_{3}+\lambda^{2} A_{2}+\lambda A_{1}+A_{0}$ of grade 3 we associate the $3 n \times 3 n$ pencil

$$
\mathcal{S}_{P}(\lambda)=\left[\begin{array}{ccc}
\lambda A_{1}+A_{0} & \lambda I & \\
\lambda I & 0 & I \\
& I & \lambda A_{3}+A_{2}
\end{array}\right],
$$

and to $P(\lambda)=\lambda^{5} A_{5}+\lambda^{4} A_{4}+\lambda^{3} A_{3}+\lambda^{2} A_{2}+\lambda A_{1}+A_{0}$ of grade 5 we associate the pencil

$$
\mathcal{S}_{P}(\lambda)=\left[\begin{array}{ccccc}
\lambda A_{1}+A_{0} & \lambda I & & & \\
\lambda I & 0 & I & & \\
& I & \lambda A_{3}+A_{2} & \lambda I & \\
& & \lambda I & 0 & I \\
& & & I & \lambda A_{5}+A_{4}
\end{array}\right] .
$$


In general, for a matrix polynomial $P(\lambda)$ of odd grade $k=2 \ell+1$, we express $P(\lambda)$ as $P(\lambda)=\lambda^{k} A_{k}+\lambda^{k-1} A_{k-1}+\widehat{P}(\lambda)$ where $\widehat{P}(\lambda)$ has odd grade $k-2=2 \ell-1$, and construct the associated block-tridiagonal $k n \times k n$ pencil $\mathcal{S}_{P}(\lambda)$ inductively by

$$
\mathcal{S}_{P}(\lambda)=\left[\begin{array}{ccc}
\mathcal{S}_{\widehat{P}}(\lambda) & & \\
& \lambda I & \\
\lambda I & 0 & I \\
& I & \lambda A_{k}+A_{k-1}
\end{array}\right] .
$$

Clearly $\mathcal{S}_{P}$ satisfies $\left(\mathcal{S}_{P}(\lambda)\right)^{T}=\mathcal{S}_{P^{T}}(\lambda)$, so $\mathcal{S}_{P}$ is a symmetric pencil whenever $P$ is a symmetric polynomial. Moreover, $\mathcal{S}_{P}$ was shown in [22] to have several additional key properties, described here in Lemma 9.3. In the rest of this section $R_{k}$ will denote the $k n \times k n$ symmetric matrix

$$
R_{k}:=\left[I_{n} \cdot I^{I_{n}}\right]
$$

Observe that premultiplying a block $k \times k$ matrix $A$ (composed of $n \times n$ blocks) by $R_{k}$ reverses the order of the block rows of $A$.

Lemma 9.3 ([22]). Let $P(\lambda)$ be any $n \times n$ matrix polynomial of odd grade $k$, regular or singular, structured or unstructured, over an arbitrary field $\mathbb{F}$ (of any characteristic). Then

(a) $\mathcal{S}_{P}(\lambda)$ is a strong linearization for $P(\lambda)$, and

(b) $R_{k} \cdot\left(\operatorname{rev}_{1} \mathcal{S}_{P}\right)=\left(\mathcal{S}_{\operatorname{rev}_{k} P}\right) \cdot R_{k}$.

Based on these properties of $\mathcal{S}_{P}$, we can now construct a $T$-palindromic strong linearization for any $T$-palindromic polynomial of odd grade. However, due to the incompatibility between the admissible elementary divisor structures of odd grade $T$-palindromic polynomials having different type (see Corollary 8.2), it is not possible to have a single uniform construction that works for both types of such polynomials. The best we can hope to do is to linearize all type +1 palindromics with a type +1 pencil, and all type -1 palindromics with a type -1 pencil. This is achieved in the next theorem. But first, we need an elementary lemma.

Lemma 9.4. Suppose that $L(\lambda)$ is a strong linearization for a polynomial $P(\lambda)$ of grade $k$. Let $\widehat{P}(\lambda):=P(-\lambda)$, and $\widehat{L}(\lambda):=L(-\lambda)$. Then $\widehat{L}$ is a strong linearization for $\widehat{P}$.

Proof. That $\widehat{L}$ is a linearization for $\widehat{P}$ follows immediately by replacing $\lambda$ by $-\lambda$ in (3.2). It remains to show that $\operatorname{rev}_{1} \widehat{L}$ is a linearization for $\operatorname{rev}_{k} \widehat{P}$. Let $L(\lambda)=\lambda X+Y$. 
Then $\operatorname{rev}_{1} L=\lambda Y+X$, and so $\left(\operatorname{rev}_{1} L\right)(-\lambda)=-\lambda Y+X$. Now $\widehat{L}(\lambda):=L(-\lambda)=$ $-\lambda X+Y$, and thus

$$
\operatorname{rev}_{1} \widehat{L}(\lambda)=\lambda Y-X=-\left(\operatorname{rev}_{1} L\right)(-\lambda) .
$$

Next, $\operatorname{rev}_{k} \widehat{P}(\lambda)=\lambda^{k} \widehat{P}(1 / \lambda)=\lambda^{k} P(-1 / \lambda)$, and so

$$
\left(\operatorname{rev}_{k} P\right)(-\lambda)=(-\lambda)^{k} P(-1 / \lambda)=(-1)^{k}\left(\operatorname{rev}_{k} \widehat{P}\right)(\lambda) .
$$

Since $\operatorname{rev}_{1} L$ is a linearization for $\operatorname{rev}_{k} P$, we know there exist unimodular matrices $E(\lambda)$ and $F(\lambda)$ such that

$$
E(\lambda) \cdot\left(\operatorname{rev}_{1} L\right)(\lambda) \cdot F(\lambda)=\operatorname{diag}\left(\left(\operatorname{rev}_{k} P\right)(\lambda), I_{k(n-1)}\right) .
$$

Replacing $\lambda$ by $-\lambda$ and using (9.2) and (9.3) we obtain

$$
-E(-\lambda) \cdot\left(\operatorname{rev}_{1} \widehat{L}\right)(\lambda) \cdot F(-\lambda)=\operatorname{diag}\left((-1)^{k}\left(\operatorname{rev}_{k} \widehat{P}\right)(\lambda), I_{k(n-1)}\right) .
$$

It follows that $\operatorname{rev}_{1} \widehat{L}$ is a linearization for $\operatorname{rev}_{k} \widehat{P}$ by multiplying both sides by the constant matrix $\operatorname{diag}\left((-1)^{k} I_{n}, I_{k(n-1)}\right)$, and using the fact that $E(-\lambda)$ and $F(-\lambda)$ are unimodular.

TheOREM 9.5 (Existence of palindromic linearizations - odd grade case). Suppose $P(\lambda)$ is a T-palindromic polynomial with odd grade $k$, regular or singular, over an arbitrary field $\mathbb{F}$ with char $\mathbb{F} \neq 2$.

(a) If $\varepsilon(P)=+1$, then the pencil $L_{P}(\lambda):=R_{k} \mathcal{S}_{P}(\lambda)$ is a strong linearization for $P$ that is also $T$-palindromic of type +1 .

(b) If $\varepsilon(P)=-1$, let $Q(\lambda):=P(-\lambda)$. Then the pencil $\widehat{L}_{P}(\lambda):=L_{Q}(-\lambda)=$ $R_{k} \mathcal{S}_{Q}(-\lambda)$ is a strong linearization for $P$ that is T-palindromic of type -1 .

Proof. (a): That $L_{P}(\lambda):=R_{k} \mathcal{S}_{P}(\lambda)$ is a strong linearization for $P$ follows immediately from the fact that $L_{P}$ is strictly equivalent to the strong linearization $\mathcal{S}_{P}$. We then have

$$
\operatorname{rev}_{1} L_{P}(\lambda)=\operatorname{rev}_{1}\left(R_{k} \mathcal{S}_{P}(\lambda)\right)=R_{k}\left(\operatorname{rev}_{1} \mathcal{S}_{P}\right)(\lambda)=\mathcal{S}_{\operatorname{rev}_{k} P}(\lambda) R_{k}
$$

by Lemma $9.3(\mathrm{~b})$. But $P$ is palindromic of type +1 , so we may continue the calculation

$$
\mathcal{S}_{\mathrm{rev}_{k} P}(\lambda) R_{k}=\mathcal{S}_{P^{T}}(\lambda) R_{k}=\left(\mathcal{S}_{P}(\lambda)\right)^{T} R_{k}=\left(R_{k} \mathcal{S}_{P}(\lambda)\right)^{T}=L_{P}^{T}(\lambda) .
$$

Thus $\operatorname{rev}_{1} L_{P}(\lambda)=L_{P}^{T}(\lambda)$, so $L$ is $T$-palindromic of type +1 .

(b): From Lemma 7.2(b) we know that $Q(\lambda)$ is $T$-palindromic of type +1 , so by part (a) the pencil $L_{Q}(\lambda)$ is a strong linearization for $Q(\lambda)$ that is also $T$-palindromic of type +1 . Then by Lemma $9.4, L_{Q}(-\lambda)=\widehat{L}_{P}(\lambda)$ is a strong linearization for 
$Q(-\lambda)=P(\lambda)$. Finally, $L_{Q}(-\lambda)$ is $T$-palindromic of type -1 , since $L_{Q}(\lambda)$ is of type +1 . $\mathrm{Q}$

Note that $L_{P}(\lambda):=R_{k} \mathcal{S}_{P}(\lambda)$ is just the pencil $\mathcal{S}_{P}(\lambda)$ with its block-rows "turned upside down", as illustrated by the following example for grade 5 palindromic polynomials $P$ of type +1 :

$$
L_{P}(\lambda)=\left[\begin{array}{ccccc} 
& & & I & \lambda A_{5}+A_{4} \\
& & \lambda I & 0 & I \\
& I & \lambda A_{3}+A_{2} & \lambda I & \\
\lambda I & 0 & I & & \\
\lambda A_{1}+A_{0} & \lambda I & & &
\end{array}\right] .
$$

The corresponding example for grade 5 palindromic polynomials $P$ of type -1 from part(b) of Theorem 9.5 is

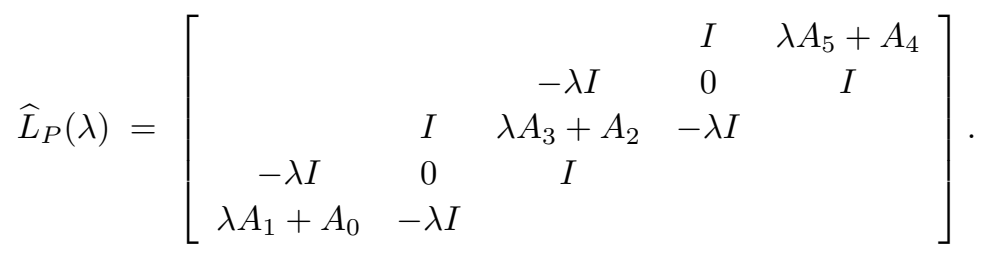

REMARK 9.6. A larger family of $T$-palindromic strong linearizations for $T$ palindromic polynomials of odd grade, distinct from the linearizations developed in this section, is constructed in [5]. These palindromic linearizations are based on the Fiedler pencils and the analysis of their properties given in [4].

Remark 9.7. It is not difficult to show that the pencil $L_{P}(\lambda)$ defined in Theorem 9.5 is $*$-palindromic whenever $P$ is, and thus provides a structured strong linearization for any $*$-palindromic matrix polynomial of odd grade.

9.2. The even grade case. The results of Section 9.1 show that every $T$ palindromic polynomial $P$ of odd grade has a strong $T$-palindromic linearization, regardless of whether $P$ is regular or singular, and irrespective of the underlying field $\mathbb{F}$. By contrast, there are several obstructions that may prevent a $T$-palindromic polynomial $P$ of even grade from having any $T$-palindromic linearization at all. One such obstruction arises from the possible incompatibility between the elementary divisor structures of $P$ and any $T$-palindromic pencil, as described in Corollary 8.2 and illustrated in Example 9.2. A completely different type of obstruction can occur when $P$ is singular, relating to the special structure of the minimal indices of singular $T$-palindromic polynomials. Since the Smith form contains no information about the minimal indices of a singular polynomial, this issue is outside the scope of this paper. Finally, there is the question of whether the underlying field $\mathbb{F}$ plays any role 
in the existence or non-existence of $T$-palindromic linearizations. The complete story, addressing all these issues, is currently under investigation. We are, however, in a position to establish results for the important special cases when $\mathbb{F}=\mathbb{R}$ or $\mathbb{F}=\mathbb{C}$ and the polynomial $P$ is regular.

THEOREM 9.8 (Existence of palindromic linearizations - even grade case). Suppose that $P(\lambda)$ is a regular T-palindromic matrix polynomial of even grade $k \geq 2$, over the field $\mathbb{F}=\mathbb{R}$ or $\mathbb{F}=\mathbb{C}$. Then $P(\lambda)$ has a strong $T$-palindromic linearization if and only if the elementary divisors of $P$ at $\lambda_{0}=+1$ and at $\lambda_{0}=-1$ satisfy the conditions described in Corollary 8.3 for either the elementary divisors of a $T$ palindromic pencil of type +1 or for one of type -1 .

Proof. The necessity of the conditions given in Corollary 8.3 is just a special case of Theorem 9.1.

To prove sufficiency, we start with a regular matrix polynomial $P(\lambda)$ whose set $\mathcal{D}$ of finite and infinite elementary divisors satisfy the conditions in Corollary 8.1 and Corollary 8.3. From the canonical forms given in $[25,26]$, it follows that there exists a $T$-palindromic regular matrix pencil having $\mathcal{D}$ as its set of elementary divisors. (Here, the elementary divisors are allowed to be complex when the polynomial $P$ is real, but the canonical form is nevertheless a real pencil.) Now by Lemma 2.3 of [3], a regular pencil $L(\lambda)$ is a strong linearization of a regular $P(\lambda)$ if and only if $L(\lambda)$ and $P(\lambda)$ have the same finite and infinite elementary divisors. Thus sufficiency is proved.

We now see that the necessary condition of the compatibility of Jordan structures described in Theorem 9.1 is, at least in the case of regular real and complex $T$ palindromic polynomials, also sufficient to guarantee the existence of a structured strong linearization. Establishing necessary and sufficient conditions for the case of general $T$-palindromic polynomials of even grade over an arbitrary field remains an open problem.

10. Conclusions. We have extended the theory of palindromic matrix polynomials with new results that apply to singular as well as regular polynomials over arbitrary fields. Zero leading coefficients have been accommodated by introducing the grade of a polynomial. The notion of reversal has been broadened, and the class of palindromic polynomials has been expanded from the one introduced in $[20,21]$.

Given any $T$-palindromic matrix polynomial, we have determined the conditions that its Smith form must satisfy: all the invariant polynomials are palindromic, and certain subsequences of the partial multiplicity sequences of the eigenvalues +1 and -1 have a particular pattern of repetition. This result allows us to characterize the possible Jordan structures of $T$-palindromic matrix polynomials, which in turn yields necessary conditions for the existence of $T$-palindromic linearizations. When the grade 
of the polynomial is odd, these conditions are shown to be sufficient as well.

\section{Appendix A. Palindromic matrix polynomials when $\operatorname{char} \mathbb{F}=2$.}

Can results analogous to those in the char $\mathbb{F} \neq 2$ case be established for $T$ palindromic polynomials over a field of characteristic two? To begin with, in any such field we have $-1=+1$, so there are no longer two different types of $T$-palindromicity, and thus, we are led to the following simplified definition.

Definition A.1 (Palindromic polynomial when char $\mathbb{F}=2$ ). A nonzero $n \times n$ matrix polynomial $P$ of degree $d \geq 0$ over a field $\mathbb{F}$ with $\operatorname{char} \mathbb{F}=2$ is said to be $T$-palindromic if $\left(\operatorname{rev}_{j} P\right)(\lambda)=P^{T}(\lambda)$ for some integer $j$, with $j \geq d$.

Almost all the results developed in Sections 4, 5, 6, and 7 as the means to prove the P-Smith form in Theorem 7.6 for char $\mathbb{F} \neq 2$ remain valid when $\operatorname{char} \mathbb{F}=2$ (deleting the statements about palindromic type). The exceptions are Lemmas 5.6 and 5.7, and Theorem 5.8, but fortunately suitable replacements can be formulated; these are presented in Lemma A.2, Lemma A.3, and Theorem A.4, respectively. This gives us all that is needed to prove the char $\mathbb{F}=2$ analog of the P-Smith form in Theorem A.5.

Lemma A.2 (Presence of $(x+1)$ factors). Let $q \in \mathbb{F}[x]$ be a nonzero $j$-palindromic polynomial, where char $\mathbb{F}=2$. If $j$ is odd, then $(x+1)$ is a factor of $q$. If $q$ is irreducible with $\operatorname{deg}(q) \geq 2$, then $q$ has even degree.

Proof. If $j$ is odd, we may write $j=2 m+1$ and express the $j$-palindromic polynomial $q$ in the form

$$
q(x)=a_{0} x^{j}+a_{1} x^{j-1}+\cdots+a_{m} x^{j-m}+a_{m} x^{m}+a_{m-1} x^{m-1}+\cdots+a_{1} x+a_{0} .
$$

Then clearly $q(1)=2\left(a_{0}+a_{1}+\cdots+a_{m}\right)$, or equivalently $q(1)=0$ since char $\mathbb{F}=2$, and so $(x+1)$ must be a factor of $q(x)$.

Now if $q$ is irreducible with $\operatorname{deg}(q) \geq 2$, then $q(0) \neq 0$, so $j=\operatorname{deg} q$. We have just shown that a polynomial with an odd grade of palindromicity cannot be irreducible. Hence $\operatorname{deg} q$ must be even.

Lemma A.3 (Multiplicity of $(x+1)$ factors). Let $p(x) \in \mathbb{F}[x]$ be a nonzero $j$-palindromic polynomial, where char $\mathbb{F}=2$. Then the multiplicity of $(x+1)$ as a factor of $p(x)$ has the same parity as $j$.

Proof. The polynomial $p(x)$ may be expressed as $p(x)=(x+1)^{\alpha} r(x)$, where $r(1) \neq 0$. Then by Lemma 5.3(c) we know that $r(x)$ is $(j-\alpha)$-palindromic. However, since $(x+1)$ is not a factor of $r(x)$, Lemma A.2 implies that $(j-\alpha)$ must be even, and hence $j$ and $\alpha$ have the same parity. 
TheOREM A.4 (Irreducible palindromic factorization in characteristic 2). A polynomial $p(x)$ over a field $\mathbb{F}$ with char $\mathbb{F}=2$ is palindromic if and only if it admits an irreducible factorization in $\mathbb{F}[x]$ of the form

$$
p(x)=c_{0} \cdot x^{k_{1}}(x+1)^{k_{2}} \cdot \prod_{i=1}^{\mu} b_{i}(x)^{m_{i}} \cdot \prod_{j=1}^{\kappa}\left(c_{j} d_{j}(x) \operatorname{rev} d_{j}(x)\right)^{n_{j}}
$$

where $c_{0}, c_{1}, \ldots, c_{\kappa} \in \mathbb{F}$ are nonzero, $k_{1}, k_{2} \in \mathbb{N}, m_{1}, \ldots, m_{\mu}, n_{1}, \ldots, n_{\kappa} \in \mathbb{N}$ are nonzero, and the irreducible factors $b_{i}(x), d_{j}(x)$ and $\operatorname{rev} d_{j}(x)$ are distinct with the following properties:

(a) Each $b_{i}(x)$ and each $d_{j}(x)$ is monic with $b_{i}(0), b_{i}(1), d_{j}(0), d_{j}(1)$ all nonzero. The nonzero constants $c_{j} \in \mathbb{F}$ are chosen so that $c_{j} \operatorname{rev} d_{j}(x)$ is also monic, for $j=1, \ldots, \kappa$.

(b) Each $b_{i}(x)$ has even degree $\operatorname{deg} b_{i} \geq 2$, and is palindromic with respect to degree.

(c) The factors $d_{j}(x)$ and $\operatorname{rev} d_{j}(x)$ are not palindromic, and may have any degree with $\operatorname{deg} d_{j}=\operatorname{deg} \operatorname{rev} d_{j} \geq 1$.

The parity of $k_{2}$ is as stated in Lemma A.3. The factorization (A.1) is unique up to reordering of the $b_{i}(x)$ 's amongst themselves, the $d_{j}(x)$ 's amongst themselves, and exchanging the roles of the monic irreducibles $d_{j}(x)$ and $c_{j} \operatorname{rev}_{j}(x)$ within each $d_{j}(x)$, $\operatorname{rev}_{j}(x)$ pair.

Proof. The proof is essentially just a simplified version of the proof of Theorem 5.8. No new ideas are required, but one needs to use Lemma A.2 (for char $\mathbb{F}=2$ ) in place of Lemma 5.6(a) (for char $\mathbb{F} \neq 2$ ).

Observe how the irreducible factorization in Theorem 5.8 simplifies when going to the char $\mathbb{F}=2$ version in Theorem A.4. Effectively, the $(x-1)$ factors get absorbed into the $(x+1)$ factors, since $-1=+1$.

With these preliminaries in hand, we can now establish the analog of the P-Smith form (Theorem 7.6) for $T$-palindromic polynomials over fields of characteristic two. Again we see that the result is simpler in the char $\mathbb{F}=2$ case.

Theorem A.5 (P-Smith form when char $\mathbb{F}=2$ ). Let $P(\lambda)$ be an $n \times n T$ palindromic matrix polynomial of grade $k$ and degree at least 1 , over a field $\mathbb{F}$ with char $\mathbb{F}=2$. Suppose that the Smith form of $P(\lambda)$ is expressed as

$$
D(\lambda)=\operatorname{diag}\left((\lambda+1)^{\beta_{1}} p_{1}(\lambda), \ldots,(\lambda+1)^{\beta_{\ell}} p_{\ell}(\lambda), 0, \ldots, 0\right)
$$

where $0 \leq \beta_{1} \leq \cdots \leq \beta_{\ell}$ are nonnegative integers, $p_{j}(\lambda)$ is monic with $p_{j}(1) \neq 0$ for $j=1, \ldots, \ell$, and $p_{j}(\lambda) \mid p_{j+1}(\lambda)$ for $j=1, \ldots, \ell-1$. Then the following hold. 
(1) $p_{j}(\lambda)$ is palindromic for $j=1, \ldots, \ell$.

(2) The subsequence consisting of all $\beta_{i}$ 's with parity opposite to that of the grade $k$ has the pairing property $\Phi$. In other words, the pairing property $\Phi$ holds for the following partial multiplicity subsequences of $\left(\beta_{1}, \beta_{2}, \ldots, \beta_{\ell}\right)$ :

\begin{tabular}{|c|c|}
\hline $\begin{array}{c}\text { Grade } k \\
\text { of } P(\lambda)\end{array}$ & $\begin{array}{l}\text { Partial multiplicity subsequences } \\
\text { of } P \text { at } \lambda_{0}=1 \text { with property } \Phi\end{array}$ \\
\hline \hline even & All odd $\beta_{j}$ 's \\
\hline odd & All even $\beta_{j}$ 's \\
\hline
\end{tabular}

We emphasize that the phrase "all even $\beta_{j}$ 's" includes any and all occurrences of 0 .

Proof. (1): The palindromicity of each $p_{j}(\lambda)$ follows from Proposition 7.1 and Lemma 5.3(c), both of which hold without change when char $\mathbb{F}=2$.

(2): We follow a two-part strategy analogous to that used in the proof of (2) in Theorem 7.6, making some important adaptations to handle the characteristic 2 case.

Step 1: Show that if $\beta_{1}$ and $k$ have opposite parity, then $n \geq 2, \ell \geq 2$, and $\beta_{2}=\beta_{1}$. Since each diagonal entry $P(\lambda)_{j j}$ is $k$-palindromic, the multiplicities of the $(\lambda+1)$ factors occurring in every $P(\lambda)_{j j}$ are of the same parity as $k$, by Lemma A.3. But $P(\lambda)_{j j}$ is divisible by $d_{1}(\lambda)$, so $(\lambda+1)$ must occur as a factor of each diagonal entry at least $\beta_{1}+1$ times. We can therefore write $P(\lambda)_{j j}=(\lambda+1)^{\beta_{1}+1} r_{j}(\lambda)$, where $r_{j}$ is palindromic. Since $d_{1}(\lambda)=(\lambda+1)^{\beta_{1}} p_{1}(\lambda)$ is the GCD of all the entries of $P(\lambda)$, this implies that there must exist some off-diagonal entry $P(\lambda)_{i j}=(\lambda+1)^{\beta_{1}} s(\lambda)$ with $i>j$ and $s(1) \neq 0$, and hence $n \geq 2$. Letting $\eta=\{i, j\}$, a calculation analogous to that in Step 1 of the proof of Theorem 7.6 yields

$$
\operatorname{det} P(\lambda)_{\eta \eta}=(\lambda+1)^{2 \beta_{1}}[\underbrace{(\lambda+1)^{2} r_{i}(\lambda) r_{j}(\lambda)+\lambda^{k-\beta_{1}} s(\lambda) s(1 / \lambda)}_{=: v(\lambda)}]
$$

in place of (7.2). We see that $v(\lambda)$ is a nonzero polynomial, since $s(1) \neq 0$ implies that $v(1) \neq 0$. Hence $\operatorname{det} P(\lambda)_{\eta \eta}$ is a nonzero polynomial, and the rest of the argument in Step 1 of Theorem 7.6 shows, mutatis mutandis, that $\ell \geq 2$ and $\beta_{2}=\beta_{1}$. (Note that when $k$ is odd, $\beta_{1}$ may be zero. The proof of Step 1 remains valid, yielding $\beta_{2}=\beta_{1}=0$ in this case.)

Step 2: Push forward down the diagonal.

Let $h_{1}<h_{2}<\cdots<h_{\nu}$ be all the positions on the diagonal where the parity of the exponent $\beta_{h_{i}}$ is opposite to that of the grade $k$, i.e., where $\beta_{h_{i}}$ is odd if $k$ is even, or $\beta_{h_{i}}$ is even if $k$ is odd. As in the proof of Step 2 in Theorem 7.6, we assume 
for purposes of contradiction that the pairing property $\Phi$ fails for the subsequence $\left(\beta_{h_{1}}, \beta_{h_{2}}, \ldots, \beta_{h_{\nu}}\right)$, and let $r:=h_{2 j-1} \leq n$ be the first position on the diagonal of $D(\lambda)$ where $\Phi$ fails. We now argue that $r<n$. There are certainly $2 j-1$ positions $\left(h_{1}, h_{2}, \ldots, h_{2 j-1}\right)$ where the factor $(\lambda+1)$ has exponent $\beta_{i}$ whose parity is opposite to $k$. If $r=h_{2 j-1}=n$, then there would be exactly $n-(2 j-1)$ positions where the exponent $\beta_{i}$ has the same parity as $k$, and therefore the total number $\gamma$ of $(\lambda+1)$ factors in $\operatorname{det} D(\lambda)$, and hence also in $\operatorname{det} P(\lambda)$, would satisfy

$$
\begin{aligned}
\gamma & \equiv(2 j-1)(k+1)+(n-2 j+1)(k) \bmod 2 \\
& \equiv(1)(k+1)+(n+1)(k) \bmod 2 \\
& \equiv(k n+1) \bmod 2
\end{aligned}
$$

which is opposite in parity to $k n$. This contradicts Lemma A.3, since by Proposition 6.1 , det $P(\lambda)$ is $k n$-palindromic. Hence $r<n$.

Next, consider the $r$ th compound matrix $\mathcal{C}_{r}[P(\lambda)]$ and its first two invariant polynomials given from Theorem 2.6 by

$$
c_{1}(\lambda)=d_{1}(\lambda) \cdots d_{r-1}(\lambda) d_{r}(\lambda) \quad \text { and } \quad c_{2}(\lambda)=d_{1}(\lambda) \cdots d_{r-1}(\lambda) d_{r+1}(\lambda) .
$$

Another mod 2 count establishes that the total number $\widehat{\gamma}$ of $(\lambda+1)$ factors in $c_{1}(\lambda)$ has opposite parity to $k r$ :

$$
\widehat{\gamma} \equiv(2 j-1)(k+1)+(r-2 j+1)(k) \equiv(k r+1) \bmod 2 .
$$

Since $\mathcal{C}_{r}[P(\lambda)]$ is $T$-palindromic of grade $k r$ by Theorem 4.6, we may invoke the result of Step 1 to conclude that $c_{2}(\lambda)$ must also be nonzero, with the same number of $(\lambda+1)$ factors as $c_{1}(\lambda)$. The desired result now follows from the same closing argument that was employed in Step 2 of Theorem 7.6.

Finally, it should be noted that the construction of Theorem 9.5(a) is still valid when char $\mathbb{F}=2$, and thus provides a $T$-palindromic strong linearization for any $T$ palindromic polynomial of odd grade, even for $T$-palindromic polynomials over a field of characteristic two.

\section{REFERENCES}

[1] E.N. Antoniou and S. Vologiannidis. A new family of companion forms of polynomial matrices. Electron. J. Linear Algebra, 11:78-87, 2004.

[2] R. Byers, D.S. Mackey, V. Mehrmann, and H. Xu. Symplectic, BVD, and palindromic approaches to discrete-time control problems. In P. Petkov and N. Christov, editors, $A$ Collection of Papers Dedicated to the 60th Anniversary of Mihail Konstantinov, Publ. House Rodina, Sofia, Bulgaria, 81-102, 2009. Also available as MIMS EPrint 2008.35, Manchester Institute for Mathematical Sciences, The University of Manchester, UK, 2008. 
[3] F. De Terán, F. Dopico, and D.S. Mackey. Linearizations of singular matrix polynomials and the recovery of minimal indices. Electron. J. Linear Algebra, 18:371-402, 2009.

[4] F. De Terán, F. Dopico, and D.S. Mackey. Fiedler companion linearizations and the recovery of minimal indices. SIAM J. Matrix Anal. Appl., 31(4):2181-2204, 2010.

[5] F. De Terán, F. Dopico, and D.S. Mackey. Structured linearizations for palindromic matrix polynomials of odd degree. MIMS EPrint 2010.33, Manchester Institute for Mathematical Sciences, The University of Manchester, UK, 2010.

[6] G. Frobenius. Theorie der linearen Formen mit ganzen Coefficienten. J. Reine Angew. Math. (Crelle), 86:146-208, 1878.

[7] F.R. Gantmacher. Theory of Matrices, volume 1. Chelsea, New York, 1959.

[8] I. Gohberg, M.A. Kaashoek, and P. Lancaster. General theory of regular matrix polynomials and band Toeplitz operators. Integral Equations Operator Theory, 11(6):776-882, 1988.

[9] I. Gohberg, P. Lancaster, and L. Rodman. Matrix Polynomials. Academic Press, New York, 1982.

[10] N.J. Higham, F. Tisseur, and P.M. Van Dooren. Detecting a definite Hermitian pair and a hyperbolic or elliptic quadratic eigenvalue problem, and associated nearness problems. Linear Algebra Appl., 351-352:455-474, 2002.

[11] A. Hilliges, C. Mehl, and V. Mehrmann. On the solution of palindromic eigenvalue problems. In Proceedings of the 4 th European Congress on Computational Methods in Applied Sciences and Engineering (ECCOMAS). Jyväskylä, Finland, 2004. CD-ROM.

[12] M. Hofer, N. Finger, J. Schöberl, S. Zaglmayr, U. Langer, and R. Lerch. Finite element simulation of wave propagation in periodic piezoelectric SAW structures. IEEE Transactions on UFFC, 53:1192-1201, 2006.

[13] R.A. Horn and C.R. Johnson. Matrix Analysis. Cambridge University Press, Cambridge, 1985.

[14] R.A. Horn and V.V. Sergeichuk. Congruences of a square matrix and its transpose. Linear Algebra Appl., 389:347-353, 2004.

[15] D. Kalman. Uncommon Mathematical Excursions: Polynomia and Related Realms, volume 35 of Dolciani Mathematical Expositions. Mathematical Association of America, Washington, D.C., 2009.

[16] P. Lancaster. Linearization of regular matrix polynomials. Electron. J. Linear Algebra, 17:21$27,2008$.

[17] P. Lancaster, U. Prells, and L. Rodman. Canonical structures for palindromic matrix polynomials. Oper. Matrices, 1:469-489, 2007.

[18] P. Lancaster and P. Psarrakos. A note on weak and strong linearizations of regular matrix polynomials. MIMS EPrint 2006.72, Manchester Institute for Mathematical Sciences, The University of Manchester, UK, 2005.

[19] P. Lancaster and M. Tismenetsky. The Theory of Matrices, 2nd edition. Academic Press, Orlando, 1985.

[20] D.S. Mackey, N. Mackey, C. Mehl, and V. Mehrmann. Palindromic polynomial eigenvalue problems: Good vibrations from good linearizations. Technical Report 239, DFG Research Center Matheon, Mathematics for key technologies in Berlin, TU Berlin, Str. des 17. Juni 136, D-10623 Berlin, Germany, 2005. Available at url: http://www.matheon.de/.

[21] D.S. Mackey, N. Mackey, C. Mehl, and V. Mehrmann. Structured polynomial eigenvalue problems: Good vibrations from good linearizations. SIAM J. Matrix Anal. Appl., 28(4):10291051, 2006.

[22] D.S. Mackey, N. Mackey, C. Mehl, and V. Mehrmann. Jordan structures of alternating matrix polynomials. Linear Algebra Appl., 432(4):867-891, 2010.

[23] M. Marcus and H. Minc. A Survey of Matrix Theory and Matrix Inequalities. Allyn and Bacon, Boston, 1964

[24] V.V. Prasolov. Problems and Theorems in Linear Algebra. American Mathematical Society, Providence, 1994 
[25] C. Schröder. A canonical form for palindromic pencils and palindromic factorizations. Technical Report 316, DFG Research Center Matheon, Mathematics for key technologies in Berlin, TU Berlin, Str. des 17. Juni 136, D-10623 Berlin, Germany, 2006. Available at url: http://www.matheon.de/.

[26] C. Schröder. Palindromic and Even Eigenvalue Problems - Analysis and Numerical Methods. PhD Thesis, Technical University of Berlin, Germany, 2008.

[27] P.G. Wodehouse. Leave it to Psmith. Herbert Jenkins, 1923.

[28] S. Zaglmayr. Eigenvalue problems in SAW-filter simulations. Diplomarbeit, Inst. of Comp. Mathematics, J. Kepler Univ. Linz, Austria, 2002. 